\title{
Radial mixing in protoplanetary accretion disks
}

\section{Metamorphosis of the silicate dust complex}

\begin{abstract}
H.-P. Gail ${ }^{\star}$
Institut für Theoretische Astrophysik, Universität Heidelberg, Tiergartenstraße 15, 69121 Heidelberg, Germany

Received 4 December 2002 / Accepted 29 August 2003

Abstract. The outer regions of protoplanetary accretion discs are formed by material from the parent molecular cloud of the freshly forming stars. The interstellar dust in this material is a mixture of species which does not correspond to any kind of chemical equilibrium state between the solid and gaseous phases. Mass accretion carries this material into the warm inner disc zones where chemical and physical processes are activated which convert the non-equilibrium solid-gas mixture into a chemical equilibrium mixture. Part of the equilibrated material is then mixed outwards by turbulent diffusion and large-scale circulation currents. This work specifically considers the evolution of the main dust components, viz. from the interstellar mixture of amorphous $\mathrm{Mg}$-Fe-silicates, into a chemical equilibrium mixture of crystalline $\mathrm{Mg}$-silicates, and iron. The basic set of equations for calculating the evolution of a mixture of silicates and iron is derived. Model calculations based on stationary, one-zone $\alpha$-discs are combined with the advection-diffusion-reaction equations for the dust evolution to study the interstellar to equilibrium dust conversion and the radial mixing of equilibrated dust into the outer disc regions. This determines the mixture of the main dust components which form the mineral inventory of planetesimals. It is found that the results of the model calculation for the resulting mineral mixture are in rough agreement with the composition of matrix material of primitive meteorites and dust in cometary nuclei.
\end{abstract}

Key words. accretion disks - dust - solar system: formation

\section{Introduction}

Observations of dust from cometary nuclei and the chemical and isotopic composition of meteoritic material from our Solar System indicate that the material of the protoplanetary accretion disc out of which our planetary system formed 4.6 Gyrs ago was subject to extensive mixing and chemical processing. Chemical and physical processing at elevated temperatures has converted the dust mixture, originating from interstellar space and from many different circumstellar environments, from a mixture with composition far from any chemical equilibrium state and with an amorphous lattice structure into a chemical equilibrium mixture of crystalline dust components. Radial mixing processes have transported such material into outer disc regions where the mixture of pristine dust from the parent molecular cloud and of equilibrated dust from warm inner disc regions is incorporated first into planetesimals and later into their descendants, the meteoritic parent bodies and the planets.

An understanding of the basic processes which are responsible for the conversion of the pristine dust mixture into a chemical equilibrium dust mixture is important in two respects.

On the one hand, the composition of cometary nuclei material and of the matrix material of primitive meteorites is

* e-mail: gail@ita.uni-heidelberg.de determined to a large extent by these processes, though considerable further processing of meteoritic matter has occurred on the surface of their parent bodies. If one attempts to understand the composition of dust in comets and of the matrix material of primitive meteorites one has to model the processes responsible for the dust metamorphosis in the early protoplanetary accretion disc.

On the other hand, dust mixtures of interstellar origin and chemical equilibrium mineral mixtures have quite different extinction properties. The composition and extinction properties of the dust component of the disc material are important for the structure and evolution of protoplanetary discs since the dust extinction determines the temperature structure in the disc, the presence or absence of convection, the shielding of the interior of the disc from ionizing radiation from the outside and so on. Modeling of the structure and evolution of protostellar and protoplanetary discs, their chemical evolution, and the determination of the raw material from which planetesimals and planets later on are formed in the disc requires a knowledge that is as precise as possible of the composition and structure of the dust.

Radial mixing processes and annealing of amorphous dust have already been treated in our previous papers (Gail 2001; Wehrstedt \& Gail 2002, henceforth called Papers I and II, respectively; Wehrstedt \& Gail 2003) and by Bockelée-Morvan et al. (2002). These model calculations showed that radial 
mixing of annealed dust from warm inner into cool outer disc regions can explain the observed fraction of crystalline dust in cometary nuclei. The present paper is a first attempt to model the processes responsible for the conversion of the interstellar dust mixture into the chemical equilibrium dust mixture dominating the inner portions of protoplanetary discs.

The true dust mixture probably is very complex; it consists of a large number of different compounds made up of elements heavier than $\mathrm{He}$, mainly compounds of the rock-forming elements $\mathrm{O}, \mathrm{Si}, \mathrm{Fe}, \mathrm{Mg}, \mathrm{Al}, \mathrm{Ca}, \mathrm{Na}, \mathrm{Ni}$ and of carbonaceous compounds, but a number of minerals of the less abundant elements are also present. For reasons of cosmic element abundance the most abundant dust components are compounds of $\mathrm{Si}, \mathrm{Mg}$, $\mathrm{Fe}$, and carbon dust. They also dominate the extinction of the disc material. To construct models of protoplanetary discs it presently suffices to concentrate on these most abundant dust species, and only the metamorphosis of these dust components is considered in the present paper. Other dust components have no significant influence on the disc structure and evolution.

In the following we outline the basic problems which have to be solved to arrive at a self consistent description of the evolution of protoplanetary discs, including the chemical evolution of their mineral content during their early evolution, when the raw material for formation of planetesimals and planetary bodies is fabricated.

\section{Composition of the dust mixture}

\subsection{The initial dust mixture}

Pollack et al. (1994) discussed which types of dust, formed from the most abundant dust forming elements (i.e. C, N, O, $\mathrm{Mg}, \mathrm{Si}, \mathrm{S}$, and $\mathrm{Fe}$ ), can be expected to exist (i) in the parent molecular cloud cores from which stars are formed, and (ii) in the cool outer parts of the resulting protostellar and protoplanetary accretion disks. They arrived at the conclusion that in the cool outer parts of an accretion disk around a newly formed star there exists a multicomponent mixture of several kinds of dust species, which is dominated most likely by the few species listed in Table 1.

This table also shows the best estimate of Pollack et al. (1994) for the fraction of the key elements $\mathrm{C}, \mathrm{Mg}, \mathrm{Fe}, \mathrm{Si}$, and $\mathrm{S}$ bound into these dust materials: The elements $\mathrm{Mg}, \mathrm{Fe}$, and $\mathrm{Si}$ are assumed to be completely condensed into dust while for $\mathrm{S}$ some fraction remains in the gas-phase. The carbon is assumed to form kerogen, a complex organic material containing considerable portions of $\mathrm{H}, \mathrm{N}$, and $\mathrm{O}$ besides the carbon. This is the carbonaceous material found in the matrix of carbonaceous chondrites. A considerable fraction of carbon (about 40\%) remains in the gas-phase, mainly as CO. Solid iron and troilite are probably solid solutions with $\mathrm{Ni}$ and $\mathrm{NiS}$, respectively.

The silicates have an amorphous lattice structure since the infrared absorption bands from silicate dust in molecular clouds show no indication of the presence of crystalline silicates. The lattice structure of quartz probably also is amorphous, though from observations nothing is known about the lattice structure of a possible silica component. It has become common practice in astrophysics to name the amorphous dust
Table 1. Composition of the dust mixture in the outer regions of a protoplanetary accretion disk according to Pollack et al. (1994). The table shows the fraction of the indicated elements condensed into the different dust species. $x$ denotes the mole fraction of the pure magnesium silicate end members of the solid solution series forming the magnesium-iron-silicates called olivine-type and pyroxene-type, respectively, on the basis of their assumed chemical composition. Only those dust components of the most abundant refractory elements are considered that are the main sources of dust opacity.

\begin{tabular}{lllllll}
\hline \hline $\begin{array}{l}\text { Species } \\
\text { formula }\end{array}$ & $\mathrm{Mg}$ & $\mathrm{Fe}$ & $\mathrm{Si}$ & $\mathrm{S}$ & $\mathrm{C}$ & $x$ \\
\hline $\begin{array}{l}\text { olivine-type } \\
\mathrm{Mg}_{2 x} \mathrm{Fe}_{2(1-x)} \mathrm{SiO}_{4}\end{array}$ & 0.83 & 0.42 & 0.63 & & 0.7 \\
pyroxene-type & 0.17 & 0.09 & 0.27 & & 0.7 \\
$\quad \mathrm{Mg}_{x} \mathrm{Fe}_{1-x} \mathrm{SiO}_{3}$ & & & & & \\
quartz-type & & & 0.10 & & \\
$\quad \mathrm{SiO}_{2}$ & & & & & \\
iron & & 0.10 & & & \\
$\quad \mathrm{Fe}$ & & & & & \\
troilite & & 0.39 & & 0.75 & \\
$\quad$ FeS & & & & \\
kerogen & & & & & \\
$\quad \mathrm{CHON}$-material & & & & & \\
\hline
\end{tabular}

species of the ISM by the mineral names of crystalline compounds with the same chemical composition, though minerals by definition are crystalline. It has been proposed by Jones (2000) to discriminate in the nomenclature such substances of unclear composition and/or non-crystalline structure more clearly from true minerals by calling them for instance olivinetype instead simply olivine and reserve the mineral names for the true minerals. We will follow this suggestion.

The numbers in Table 1 result from a critical discussion and evaluation of the observational material and probably represent the most reliable model for the composition of the dust material in molecular cloud cores and in protostellar disks which can presently be derived. We base our considerations in this paper with respect to the pristine $\mathrm{Mg}-\mathrm{Fe}-\mathrm{Si}$ dust components in a protoplanetary disc precisely on this model. We shall call this the P94-mixture.

The P94 model for the dust composition is quite different from models for the interstellar dust composition, as for instance the widely used Mathis-Rumpl-Nordsieck model (Mathis et al. 1977) or the model proposed by Li \& Greenberg (1997). Especially the assumption of an iron and a troilite component is unusual. It is motivated by results of thermochemical equilibrium calculations (e.g. Grossman 1972; Lattimer et al. 1978; Prinn \& Fegley 1989) and the existence of such grains in primitive meteorites. The recent detection of iron and troilite inclusions in glassy silicate grains of presumably interstellar origin (Bradley 1994, cf. also the discussion in Martin 1995; Goodman \& Whittet 1995) and the observational result of Howk et al. (1999) lends some support to the assumptions of 


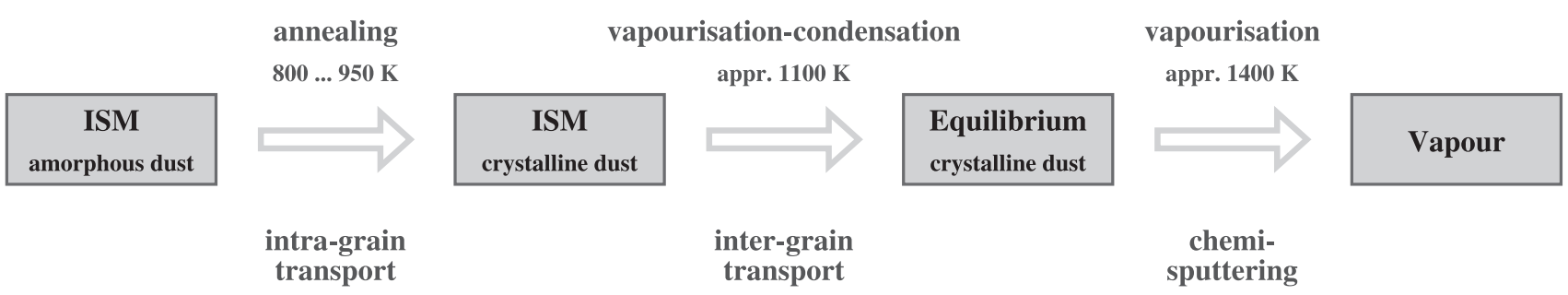

Fig. 1. Processes responsible for the conversion of the interstellar silicate dust mixture into a chemical equilibrium dust mixture and its final destruction.

the P94 model. Also the models for the composition of interstellar dust discussed by Mathis (1996) show iron grains to be a possible component of the interstellar dust mixture, and FeS recently has been detected in protoplanetary discs (Keller et al. 2002).

In the P94 model no calcium-aluminium compounds are included, though such grains of definitely interstellar origin are observed to exist in the disc material (Nittler et al. 1997; Choi et al. 1999). They do not constitute, however, abundant dust components of the dust mixture. For this reason they are not important for the opacity of the disc material in the outer disc regions, where the $\mathrm{P} 94$ mixture dominates.

One assumption of the P94 model needs a special comment: The presence of $\mathrm{SiO}_{2}$ as a separate dust component is somewhat doubtful since there are no indications for this material to be an abundant part of the interstellar dust mixture. On the other hand, theoretical calculations (Gail \& Sedlmayr 1999) indicate that silica may be formed as a minor dust component in circumstellar dust shells and then would be injected into the interstellar medium. Observationally it is found that silica seems to be present in protoplanetary discs at later evolutionary stages (Bouwman et al. 2001; Honda et al. 2003). For this reason we apply the P94 model without changes.

\subsection{Modification of the dust composition}

Viscous torques in the accretion disc induce a slow inwards migration of the disc material by which the material is transported from the outer disc into increasingly warmer zones. On the other hand, turbulent mixing in the convectively unstable parts of the disc and large scale circulation currents intermingle material from different disc regions and transport some material from the warm inner into the cold outer parts of the disc. At sufficiently high temperature, solid diffusion and annealing processes are activated in the dust grains, which then tend

- to form a regular crystalline lattice structure (Duschl et al. 1996; Gail 1998; Hallenbeck et al. 1998; Fabian et al. 2000) and

- to exsolve impurities from the grain lattice.

By these processes the grain material develops from the dirty and amorphous composition which is responsible for the observed extinction properties of grains in interstellar space and molecular clouds to a more clean and (poly)crystalline lattice structure. The chemical composition of the individual grains, however, is not changed by these processes since the inventory of chemical elements within each grain is at most redistributed within a grain but not exchanged between different grains. The whole assemblage of minerals forming the dust mixture essentially remains preserved at this stage, since there operate only intra-grain transport processes by solid diffusion, but no inter-grain transport processes via the gas-phase.

A change in composition and structure of the individual grains is often accompanied by considerable changes of their extinction properties (cf. Fig. 1 of Paper I), which in turn strongly affect the disc structure. This change of the lattice structure and of the optical properties of the dust grains occurs in the region where the timescale for solid diffusion and annealing roughly equals the timescale for the temperature increase which is of the order of the timescale of radial inwards migration of the disc material. The model calculations in Papers I and II showed this to occur at about $800 \mathrm{~K}$ for glassy silicates. If the structure of the silicate dust is similar to that of the smokes prepared in the annealing experiments of Hallenbeck et al. (1998) one determines from the data in Hallenbeck et al. (2000) a somewhat higher annealing temperature of about $950 \mathrm{~K}$ (cf. Paper I). At temperatures above the annealing temperature the grains have a crystalline lattice structure, which at the same time increases their stability against evaporation.

At a temperature significantly higher than that required for annealing, transport processes between different grains via the gas-phase start to operate by means of evaporation and re-condensation. These inter-grain transport processes via the gas-phase tend to remove the thermodynamically less stable condensed components from the interstellar mineral mixture in favour of the thermodynamically most stable minerals existing in a state characterized by

- a given pressure $P$ and temperature $T$, and

- a given elemental composition corresponding to the mean composition of the whole mixture of grains coming from the parent molecular cloud.

The basic reason for the inter-grain transport via the gas-phase is that the less stable components tend to have higher vapour pressures than the more stable components.

Since the pristine dust mixture inherited from the molecular cloud contains dust species that have been formed in such chemically diverse environments as circumstellar shells of AGB-, RSG-, WC-stars, and supernovae, or by destruction and re-condensation behind shocks in the ISM, many of the mineral components of the mixture are thermodynamically 
unstable in an element mixture corresponding to the mean element mixture of the protoplanetary disc material. The latter mixture results from mixing material from a large number of different sources with widely different element compositions. Though the dust grains from the different sources were completely stable in the special environment in which they were originally formed, many of them are unstable in the resulting mean element mixture of the protoplanetary accretion disc. Grains consisting of such unstable materials slowly vanish by evaporation or chemi-sputtering while their material is transported via the gas-phase and finally is consumed by the growth of mineral grains which are the most stable ones for the mean element mixture in the protoplanetary disc.

This process (evaporation, transport via the gas-phase, recondensation) drives the non-equilibrium mineral assemblage of the dust originating from circumstellar environments and the ISM towards an equilibrium composition corresponding to the averaged element mixture in the protoplanetary disc. Hence, the transport, diffusion, annealing, evaporation, and recondensation processes, schematically indicated in Fig. 1, result in the existence of two completely different dust mixtures in the inner and outer part of the accretion disc:

1. Amorphous dust with a strong non-equilibrium composition in the cold outer parts of the disc.

2. Crystalline dust with chemical equilibrium composition in the warm inner parts of the disc right into the region of evaporation of the solids.

A realistic calculation of the disc structure needs to include the gradual change in dust composition and structure from the P94 mixture of grains of interstellar origin encountered in the cool outer parts of the disc, into the chemical equilibrium mixture existing in the warm inner parts of the disc.

\subsection{The thermal equilibrium mineral mixture}

The regions of existence in the $P$ - $T$-plane of the different possible condensates in chemical equilibrium have often been calculated as the so-called condensation sequence for Solar System abundances (e.g. Grossman 1972; Lattimer et al. 1978; Saxena \& Ericksson 1986; Sharp \& Huebner 1990; Ebel \& Grossman 2001 and many more). Within the context of the structure of protoplanetary discs this problem is discussed in Gail (1998). From such calculations it is known that in chemical equilibrium only a few of the vast number of possible condensates really do exist. For conditions valid in a protoplanetary accretion disc the condensates formed from the abundant refractory elements $\mathrm{Si}$, $\mathrm{Mg}, \mathrm{Fe}, \mathrm{Al}$, and $\mathrm{Ca}$ are:

- the calcium-aluminium compounds:

- corundum $\left(\mathrm{Al}_{2} \mathrm{O}_{3}\right)$ or hibonite $\left(\mathrm{CaAl}_{6} \mathrm{O}_{19}\right)$;

- gehlenite $\left(\mathrm{Al}_{2} \mathrm{Ca}_{2} \mathrm{SiO}_{7}\right)$;

- spinel $\left(\mathrm{MgAl}_{2} \mathrm{O}_{4}\right)$;

- diopside $\left(\mathrm{MgCa}\left(\mathrm{SiO}_{3}\right)_{2}\right)$;

- anorthite $\left(\mathrm{CaAl}_{2} \mathrm{Si}_{2} \mathrm{O}_{8}\right)$;

- the silicate compounds:

- forsterite $\left(\mathrm{Mg}_{2} \mathrm{SiO}_{4}\right)$ and fayalite $\left(\mathrm{Fe}_{2} \mathrm{SiO}_{4}\right)$;

- enstatite $\left(\mathrm{MgSiO}_{3}\right)$ and ferrosilite $\left(\mathrm{FeSiO}_{3}\right)$;
- the iron-bearing condensates:

- metallic iron $(\mathrm{Fe})$;

- troilite $(\mathrm{FeS})$.

Solid condensates of some other, less abundant elements, do exist, but the abundances of such trace condensates, e.g. Ti-compounds, are too low for them to contribute significantly to the opacity of the disc material and, thus, are not important for the structure of protoplanetary discs. They are neglected for this reason in the present model calculation, but it should be kept in mind that because of the particular high thermal stability of some of such rare compounds they may serve as growth centres for less stable dust grains formed from more abundant elements.

Many of the condensates form solid solutions with other solid compounds. This especially holds for

- metallic iron, which takes up all the available $\mathrm{Ni}$,

- forsterite $\left(\mathrm{Mg}_{2} \mathrm{SiO}_{4}\right)$, which forms olivine, a solid solution with fayalite $\left(\mathrm{Fe}_{2} \mathrm{SiO}_{4}\right)$, and

- enstatite $\left(\mathrm{MgSiO}_{3}\right)$, which forms pyroxene, a solid solution with ferrosilite $\left(\mathrm{FeSiO}_{3}\right)$.

The secondary solution components in all cases have only small concentrations in the mixture (e.g. Saxena \& Ericksson 1986) and, for this reason, are neglected in the following calculations. The inclusion of solid solutions instead of the pure substances into a model calculation would be straightforward, but because of the lack of data for optical constants does not improve the modeling of the disc opacity.

We have calculated the abundances of molecular and solid chemical compounds of the most abundant refractory elements $\mathrm{Si}, \mathrm{Mg}, \mathrm{Fe}, \mathrm{Al}, \mathrm{Ca}$ for a Solar System element mixture (Anders \& Grevesse 1989, with corrections from Grevesse \& Noels 1993; Holweger 2001), by the methods of chemical thermodynamics. Results for the stability limits of the condensates are shown in Fig. 2. These are the most abundant solids existing in a chemical equilibrium state in the disc between $\approx 500 \mathrm{~K}$ and the upper limit of existence of dust. This mixture is formed, if the pristine P94 dust mixture evolves into chemical equilibrium by complete evaporation and re-condensation. The dotted lines show the $P$ - $T$-trajectories of the midplane of a disc model in the one-zone approximation and the corresponding trajectory for the disc photosphere at $\tau=\frac{2}{3}$ (calculated as described in Paper I).

In the present paper we concentrate on the conversion of the silicate and iron dust components of the P94 dust mixture into the chemical equilibrium mixture of forsterite, enstatite and iron, and its consequences for the disc structure. The obviously much more complicated problem of the Ca-Al-compounds is not treated for two reasons: First, dust components formed from elements with an abundance less than that of the Fe-Mg-Si complex do not contribute significantly to the opacity of the disc material, and thus do not have a significant influence on the calculated disc structure. Second, presently it is not known whether $\mathrm{Al}$ and $\mathrm{Ca}$ in the pristine interstellar dust mixture are mainly present as dust species of their own or if part or most of these elements are incorporated 


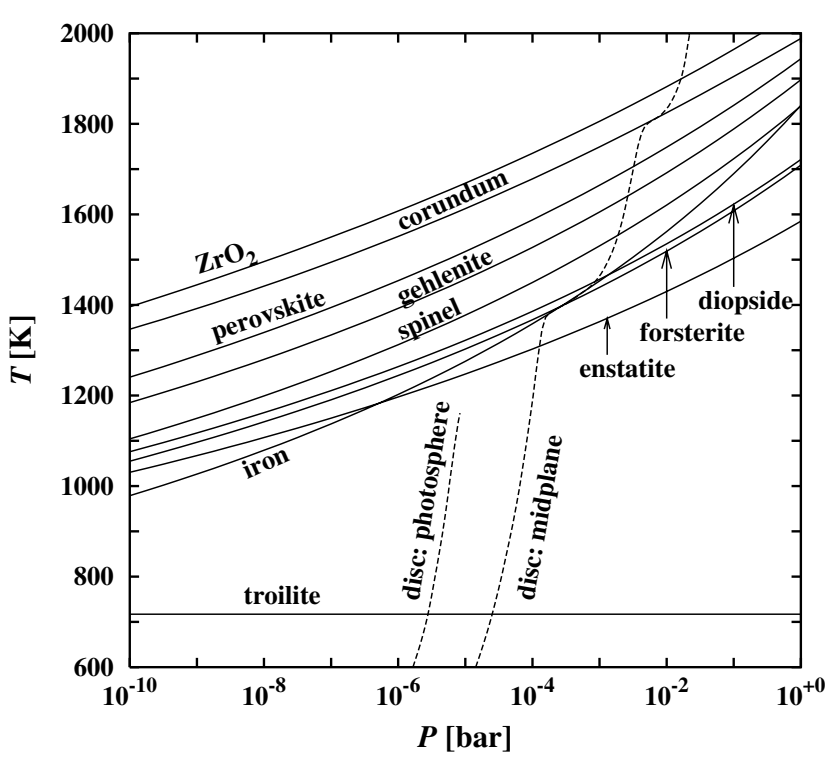

Fig. 2. Stability limits in the $P-T$ plane of the minerals formed in chemical equilibrium by the most abundant refractory elements $\mathrm{Si}$, $\mathrm{Mg}, \mathrm{Fe}, \mathrm{Al}, \mathrm{Ca}$ in a Solar System element mixture. The dashed lines are the $P$ - $T$-trajectories corresponding to the disc photosphere at $\tau=\frac{2}{3}$ (left) and the midplane of the disc (right) for a model with accretion rate $\dot{M}=10^{-7} M_{\odot} \mathrm{yr}^{-1}$.

into the more abundant $\mathrm{Mg}$-Fe-silicates. In our model calculation we consider only corundum as a representative mineral from the Ca-Al-compound complex in order to account for the dust opacity between the stability limits of the silicates and iron at about $1450 \mathrm{~K}$ and the stability of corundum at about $1800 \mathrm{~K}$, where only the $\mathrm{Ca}-\mathrm{Al}$-compounds contribute to the dust opacity.

At low temperatures some of the abundant condensates may be converted into different solid compounds, e.g. troilite and iron oxides etc. Such dust components are also outside the scope of the present paper.

\subsection{The dust mixture}

In our discussion of the conversion of the silicate-iron dust complex from its interstellar composition into a chemical equilibrium composition we consider the following dust species:

- amorphous olivine-type, amorphous pyroxene-type, and quartz-type dust of interstellar origin and their crystalline counterparts after annealing,

- iron grains,

- crystalline forsterite and crystalline enstatite formed from growth processes in the protoplanetary disc.

In the model construction of the protoplanetary disc we additionally consider carbon and corundum dust. These dust components are not related directly to the problem of the evolution of the silicate-iron dust complex but they are important for the disc structure and need to be considered in model calculations. These dust components are treated as in Paper I and therefore are not further discussed in the present paper.

\section{Important processes}

The radial distribution and the composition and properties of the various dust components in the disk are determined by a number of processes:

1. Viscous accretion slowly carries the dust particles embedded into the carrier gas inwards towards regions of higher temperature.

2. Turbulent diffusion and large scale-circulation mixes grains inwards and outwards and tends to smear out radial concentration gradients.

3. Annealing converts the amorphous dust component into crystalline dust.

4. Vaporization and chemical surface reactions erode the dust grains. The removed material increases the abundance of certain molecular species of the gas-phase.

5. Condensation processes accumulate material from the gasphase onto the surface of already existing dust particles, or new condensates may be formed by nucleation and grain growth. At the same time the gas-phase is depleted by particle growth from condensible material.

6. Solid diffusion tends to eliminate internal concentration gradients in grains with inhomogeneous composition. For instance the cations $\mathrm{Mg}^{++}$and $\mathrm{Fe}^{++}$may be distributed inhomogeneously within the silicates olivine and pyroxene at low temperatures, but their distribution will be homogenized at elevated temperatures where diffusion is activated (cf. Fig. 5).

This is just a short list of processes which we believe to be important for the evolution of the dust grain mixture in the accretion disc environment. Additional processes may be important in special circumstances or at later stages of the disc evolution, for instance the important coagulation and fragmentation processes at the onset of planetesimal formation.

The first three processes are treated in Paper I. The same method of modeling radial transport and annealing is applied in the model calculations in this paper. We will not repeat the discussion of these processes here.

The processes listed as items 4. and 5. are treated in this paper. With respect to the processes of evaporation and condensation we take advantage of the fact that annealing occurs at a temperature much lower than the temperature where evaporation of dust becomes important (cf. Papers I and II). This allows us to treat annealing as a separate process, which can be calculated independently of the processes responsible for grain destruction and grain growth, since both processes operate in different regions of the disc.

\section{The advection-diffusion-reaction equations}

\subsection{The inter-grain vapour transport}

The conversion of the non-equilibrium into the chemical equilibrium dust mixture by evaporation and re-condensation requires the transport of material through the gas-phase. This process may be limited by slow diffusion of vapour species in the gas-phase. In Gail (2003) it is shown, that vaporization is limited by diffusion only if the average grain sizes significantly 
exceed $1 \mu \mathrm{m}$. This becomes a problem if coagulation of grains starts to become efficient at the onset of the planetesimal formation process. We are mainly interested in the evolutionary phase of accretion discs which precedes the planetesimal formation process, since the essential processes determining the composition of the ingredients from which planetesimals do form operate during this phase. Diffusional limitation of intergrain vapour transport becomes important only in later phases of the disc evolution.

Additionally we assumed that the grains are at rest with respect to the gas. The more general case where grains are moving relative to the gas is considered in Gail (2003), where it is shown that this has no important effect for micron-sized grains.

\subsection{Equation for the dust grains}

In the following the chemically or physically different dust species are distinguished by a lower index $j$. For each of the substances we consider a set of fixed grain radii $a_{i}$. The number density of grains having radii falling into the radius interval $\left[a_{i}, a_{i+1}\right]$ is divided by the number density of hydrogen nuclei. The resulting quantity, the concentration of dust grains of species $j$ with radii $a_{i} \ldots a_{i+1}$ relative to hydrogen nuclei, is denoted by $c_{j, i}$. We use the same radius grid $a_{i}$ for all dust species $j$. All grains are assumed to have spherical shape.

The equation for the transport, diffusion and evaporation of dust in the one-zone approximation is derived in Paper I. The time evolution of the concentration $c_{j, i}$ of dust species $j$ with radii in $a_{i} \ldots a_{i+1}$ with respect to the total number of hydrogen nuclei is

$$
\begin{aligned}
& \frac{\partial c_{j, i}}{\partial t}+v_{\mathrm{r}} \frac{\partial c_{j, i}}{\partial r}=\frac{1}{n r} \frac{\partial}{\partial r} r n D \frac{\partial c_{j, i}}{\partial r} \\
& +\left\{\begin{array}{l}
\left(\frac{c_{j, i+1}}{\Delta a_{i+1}}-\frac{c_{j, i}}{\Delta a_{i}}\right)\left|\frac{\mathrm{d} a_{j, i}}{\mathrm{~d} t}\right| \text { if } \quad \frac{\mathrm{d} a_{j, i}}{\mathrm{~d} t}<0 \\
\left(\frac{c_{j, i-1}}{\Delta a_{i-1}}-\frac{c_{j, i}}{\Delta a_{i}}\right) \frac{\mathrm{d} a_{j, i}}{\mathrm{~d} t} \quad \text { if } \quad \frac{\mathrm{d} a_{j, i}}{\mathrm{~d} t}>0
\end{array}\right.
\end{aligned}
$$

$v_{\mathrm{r}}$ is the radial drift velocity of viscous accretion. We presently neglect any drift of the grains relative to the gas. $D$ is the diffusion coefficient of the grains induced by turbulent mixing. This is calculated in the $\alpha$-approximation as in Paper I and is assumed in this paper to be the same for all grains of species $j$ and radii $i$. The terms depending on $v_{\mathrm{r}}$ and $D$ describe the transport and mixing of the dust component in the protoplanetary disc. The smallest and the largest grain sizes in our sample require special treatment, see Paper I.

The quantity $\mathrm{d} a_{j, i} / \mathrm{dt}$ describes the change of radius of the dust grains of species $j$ and radius $a_{i}$ by growth $\left(\mathrm{d} a_{j, i} / \mathrm{dt}>0\right)$ or evaporation $\left(\mathrm{d} a_{j, i} / \mathrm{dt}<0\right)$, respectively. These terms describe size changes of grains by chemical processes, in which the grains are involved. In the limiting case that dust growth or evaporation is not diffusion limited, and that there is no relative motion between the grains and the gas, they are given by

$$
\frac{\mathrm{d} a_{j, i}}{\mathrm{~d} t}=V_{j, 0} v_{\mathrm{th}} \alpha\left(n-\frac{p_{\mathrm{eq}}}{k T}\right)
$$

Table 2. Coefficients required for calculating evaporation and conden-

\begin{tabular}{|c|c|c|c|c|}
\hline $\begin{array}{l}\text { Dust } \\
\text { species }\end{array}$ & $\begin{array}{c}\rho \\
\mathrm{g} \mathrm{cm}^{-3}\end{array}$ & $A$ & $\begin{array}{c}V_{0} \\
\mathrm{~cm}^{3}\end{array}$ & $\alpha$ \\
\hline quartz & 2.65 & 60.085 & $3.80 \times 10^{-23}$ & 0.05 \\
\hline iron & 7.87 & 55.845 & $1.19 \times 10^{-23}$ & 0.9 \\
\hline forsterite & 3.21 & 140.694 & $7.33 \times 10^{-23}$ & 0.1 \\
\hline fayalite & 4.30 & 203.774 & & \\
\hline enstatite & 3.19 & 100.389 & $5.26 \times 10^{-23}$ & 0.1 \\
\hline ferrosilite & 4.00 & 131.93 & & \\
\hline olivine & & & $7.55 \times 10^{-23}$ & 0.1 \\
\hline pyroxene & & & $5.35 \times 10^{-23}$ & 0.1 \\
\hline
\end{tabular}
sation rates. Data for $\rho$ and $A$ from the CRC-handbook (Lide 1995). For $V_{0}$ and $\alpha$ see text.

The volume $V_{j, 0}$ of the (nominal) molecule is given by

$V_{j, 0}=\frac{A_{j} m_{\mathrm{AMU}}}{\rho_{\mathrm{sol}, j}}$

where $A_{j}$ is the molecular weight and $\rho_{\mathrm{sol}, j}$ the mass density of the solid. $v_{\text {th }}$ is the root-mean-square thermal velocity of the key molecule in the evaporation process, $n$ its gas-phase particle density, and $p_{\text {eq }}$ is the partial pressure of this molecule in a chemical equilibrium state.

$\alpha$ is the evaporation coefficient respectively the growth or sticking coefficient, which are assumed to equal each other. The evaporation/sticking coefficients $\alpha$ for the silicates are discussed in Gail \& Sedlmayr (1999), Ferrarotti \& Gail (2001) and Gail (2003). The adopted values for $\alpha$ are given in Table 2.

The solution of Eq. (1) requires the prescription of boundary conditions at the inner and outer boundary of the disc model. The boundary conditions are different for the different species and are described later when we discuss the individual dust components.

\subsection{Equation for the gas-phase species}

For the gas-phase species, of primary interest are the equations for such atoms and molecules which are involved in the growth and destruction of the dust species considered in our model calculation.

The chemistry of the dust species considered involve the elements $\mathrm{O}, \mathrm{Si}, \mathrm{Fe}$, and $\mathrm{Mg}$. The gas-phase chemistry of these elements in the disc region of interest is quite simple: All oxygen not bound in the molecular species $\mathrm{CO}, \mathrm{SiO}$ and in minerals is present in the gas-phase as $\mathrm{H}_{2} \mathrm{O}$. All other O-bearing molecular compounds can be neglected. Because of the high bond energy of the $\mathrm{CO}$ molecule the $\mathrm{CO}$ abundance is fixed by the gas-phase carbon element abundance, since formation of $\mathrm{CO}$ consumes all the available carbon. Note that growth and evaporation of silicate and iron dust occur at a significantly higher temperature than carbon dust destruction which means that in the disc zone where the silicates evaporate the gasphase abundance of $\mathrm{C}$ equals the total element abundance of $\mathrm{C}$. 
$\mathrm{Mg}$ and $\mathrm{Fe}$ are present in the gas-phase as free atoms. All other molecular compounds of $\mathrm{Fe}$ and $\mathrm{Mg}$ can be neglected. At the low temperatures of interest the silicon is present in the gasphase as $\mathrm{SiO}$ and a small fraction as $\mathrm{SiS}$, all other Si-bearing compounds are negligible. For simplicity we neglect also SiS and assume that all Si present in the gas-phase is bound in $\mathrm{SiO}$.

The abundance of $\mathrm{O}$ for the Solar System element mixture exceeds the $\mathrm{Si}$ abundance by a factor of about 15 . Though some fraction of the $\mathrm{O}$ is bound in $\mathrm{CO}$ and in minerals, the rate of silicate grain growth is not limited by the $\mathrm{H}_{2} \mathrm{O}$ abundance but is limited by the abundances of $\mathrm{SiO}, \mathrm{Fe}$, and $\mathrm{Mg}$ in the gas-phase. Water vapour is always present in sufficient quantities for grain growth such that its precise abundance needs not be known in the zone of silicate dust evaporation/condensation. Then we only need to know the gas-phase abundances of $\mathrm{SiO}$ molecules and $\mathrm{Fe}$ and $\mathrm{Mg}$ atoms. These three species are not involved in any other chemical reaction of importance. Their abundances are determined by the equation for the transport and diffusion processes and by reactions with solids:

$\frac{\partial c_{m}}{\partial t}+v_{\mathrm{r}} \frac{\partial c_{m}}{\partial r}=\frac{1}{n r} \frac{\partial}{\partial r} r n D \frac{\partial c_{m}}{\partial r}+\frac{R_{m}}{N_{\mathrm{H}}}$.

$c_{m}$ is the concentration of the species $m$ (now a molecule) relative to hydrogen nuclei, $N_{\mathrm{H}}$ the number density of hydrogen nuclei and $R_{m}$ the rate of change of the number density of molecule $m$ by evaporation/condensation processes.

For each of the solids of species $j$ the rate of exchange of material between the gas-phase and the dust grains of radius $a_{i}$ has the following form:

$\frac{R_{m, j}}{N_{\mathrm{H}}}=\sum_{i} 4 \pi a_{i}^{2} c_{j, i} \frac{1}{V_{j, 0}} \frac{\mathrm{d} a_{j, i}}{\mathrm{~d} t} v_{m, j}$.

Here $4 \pi a_{i}^{2}$ is the surface area of a grain of radius $a_{i}, c_{j, i}$ the corresponding concentration of grains of species $j$ and radii between $a_{i}$ and $a_{i+1}$, the next two terms are the net rate of the number of (nominal) monomers added to or removed from the grain per unit time and surface area by chemical reactions with the gas-phase, and $v_{m, j}$ is the number of molecules of kind $m$ added to or removed from the gas-phase by the reaction between the grain and the gas-phase.

The rate term on the r.h.s of Eq. (4) is obtained by summation over the individual contributions (5) of all such reactions between the gas-phase and grains of species $j$ in which a molecule of kind $m$ is consumed or liberated. Pure gas-phase reactions can be neglected for the gas-phase species of interest ( $\mathrm{SiO}, \mathrm{Fe}, \mathrm{Mg})$.

\section{Conversion of the interstellar dust mixture into the chemical equilibrium dust mixture}

\subsection{Vapour pressures of the silicate dust species}

The silicate dust components from the parent molecular cloudiron-rich olivine-type and pyroxene-type compounds with a mole fraction of 0.3 of the iron-rich end members of the solid solution series, and quartz-type material - are not stable in an element mixture with Solar System element abundances. In chemical equilibrium $\mathrm{Mg}$ and $\mathrm{Si}$ are condensed into nearly pure forsterite $\mathrm{Mg}_{2} \mathrm{SiO}_{4}$, and enstatite $\mathrm{MgSiO}_{3}$ with only trace amounts of the iron-bearing end members $\mathrm{Fe}_{2} \mathrm{SiO}_{4}$ and $\mathrm{FeSiO}_{3}$, respectively, bound in these solids. Quartz does not even exist in chemical equilibrium at all. The evolution of the P94 dust mixture into a chemical equilibrium mixture requires the conversion of the iron-rich olivine-type, enstatite- and quartz-type ISM dust species into nearly iro-free forsterite and enstatite and into solid iron. In a protoplanetary accretion disc where the dust is suspended in a carrier gas this conversion has to occur by evaporation and re-condensation.

At the instant when the grains of ISM origin start to evaporate, their lattice structure is already converted by annealing from an amorphous to a crystalline state (cf. Paper I and Fig. 1), i.e., at the instant of their vaporization they are true olivine, pyroxene and quartz mineral grains. This makes it possible to use data for crystalline materials in model calculations of their evaporation. This point is further discussed in Sect. 5.2.

The key step for the evaporation of the silicate compounds is the ejection of a $\mathrm{SiO}$ molecule from the solid into the gasphase. For forsterite this has been checked by laboratory experiments by Nichols \& Wasserburg (1995) and Nichols et al. (1998), for enstatite this follows from the results of the laboratory experiments for enstatite evaporation of Tachibana et al. (2002), and for $\mathrm{SiO}_{2}$ from the laboratory study of Hashimoto (1990). Hence, the calculation of the change of grain radii $\mathrm{d} a / \mathrm{d} t$ by evaporation by means of Eq. (2) requires us to know the vapour pressure of the $\mathrm{SiO}$ molecules, since this determines the rate of evaporation of the silicates. For the following discussions, therefore, we have to determine the vapour pressures of $\mathrm{SiO}$ molecules for the different dust species from the $\mathrm{Si}-\mathrm{Mg}-\mathrm{Fe}$ complex.

The dust conversion process in a protoplanetary accretion disc occurs in a disc region where the pressure of the carrier gas is of the order of $10^{-4} \ldots 10^{-3}$ bar. We have calculated the chemical equilibrium composition of a gas-dust mixture with Solar System element composition for a pressure of $P=10^{-4}$ bar and the temperature region of interest between $800 \mathrm{~K}$ and $1400 \mathrm{~K}$. The resulting partial pressures of $\mathrm{Mg}$, $\mathrm{Fe}$, and $\mathrm{H}_{2} \mathrm{O}$ in chemical equilibrium between the gas-phase and the equilibrium condensates are used to calculate the chemical equilibrium vapour pressure of $\mathrm{SiO}$ for the reactions

$$
\begin{aligned}
\mathrm{Mg}_{2 x} \mathrm{Fe}_{2(1-x)} \mathrm{SiO}_{4}+3 \mathrm{H}_{2} \longleftrightarrow \\
2 x \mathrm{Mg}+2(1-x) \mathrm{Fe}+\mathrm{SiO}+3 \mathrm{H}_{2} \mathrm{O} \\
\mathrm{Mg}_{x} \mathrm{Fe}_{(1-x)} \mathrm{SiO}_{3}+2 \mathrm{H}_{2} \longleftrightarrow \\
x \mathrm{Mg}+(1-x) \mathrm{Fe}+\mathrm{SiO}+2 \mathrm{H}_{2} \mathrm{O} \\
\mathrm{SiO}_{2}+\mathrm{H}_{2} \longleftrightarrow \mathrm{SiO}+\mathrm{H}_{2} \mathrm{O} .
\end{aligned}
$$

The resulting vapour pressures of $\mathrm{SiO}$ for iron-rich olivine, pyroxene and for quartz are shown in Fig. 3 together with the partial pressure of $\mathrm{SiO}$ in equilibrium with forsterite and enstatite.

The vapour pressures of $\mathrm{SiO}$ for olivine, pyroxene, and quartz obviously are much higher, at least by a factor of three (see Fig. 3), than the vapour pressure of $\mathrm{SiO}$ for forsterite and enstatite if the partial pressures of $\mathrm{Mg}, \mathrm{Fe}$, and $\mathrm{H}_{2} \mathrm{O}$ are fixed by the presence of forsterite, enstatite, and solid iron in equilibrium with the gas-phase. The $\mathrm{SiO}, \mathrm{Mg}$, and Fe evaporated off 


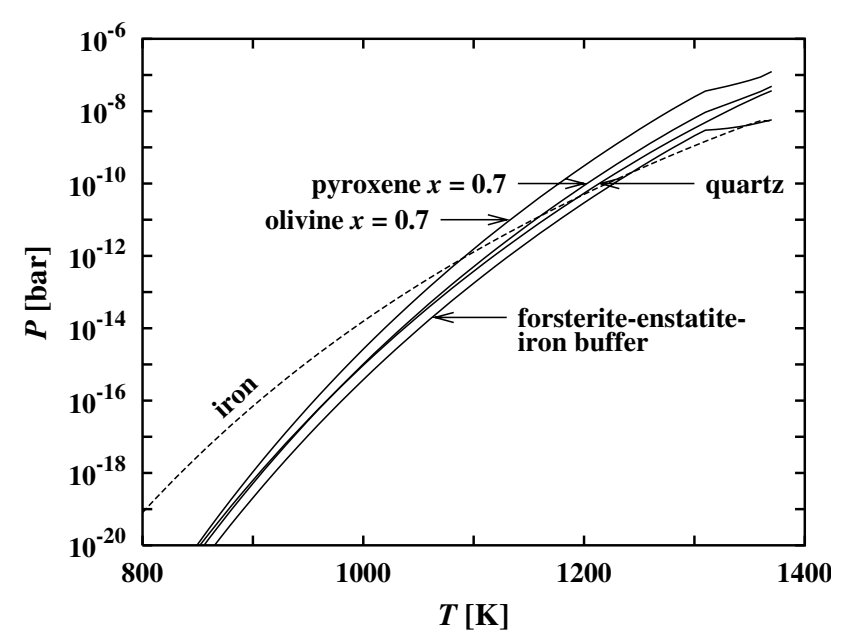

Fig. 3. Partial pressures of $\mathrm{SiO}$ in chemical equilibrium between a gas with Solar System element composition and (i) olivine and pyroxene with a mole fraction of 0.7 of the magnesium-rich end-members of the solid solution series, (ii) quartz, and (iii) the partial pressure of $\mathrm{SiO}$ of the forsterite-enstatite buffer. The total pressure is $P=10^{-4}$ bar. The dashed line shows the vapour pressure of iron.

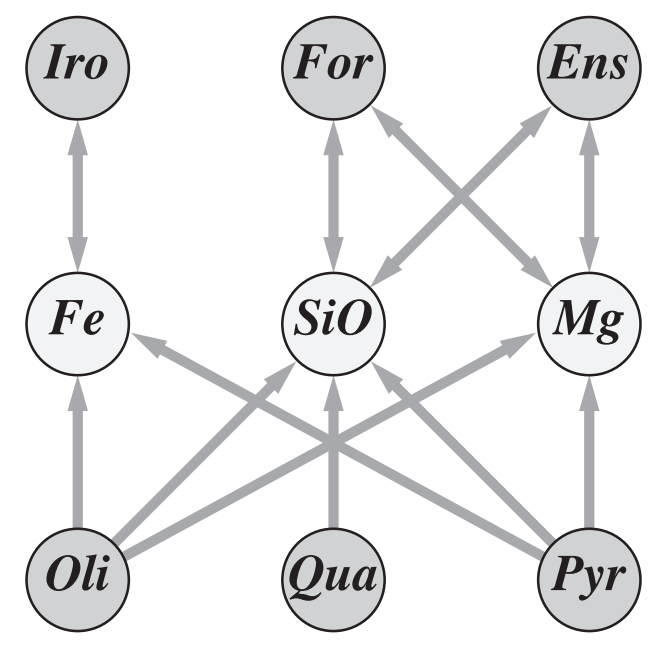

Fig. 4. Exchange of material between annealed pristine dust components with crystalline structure and equilibrium dust components via the gas-phase. The middle row refers to the vapour species. The solids in the upper and lower row are denoted by obvious abbreviations.

olivine, pyroxene, and quartz for this reason chiefly precipitate onto forsterite, enstatite, and iron and only a small fraction of the $\mathrm{SiO}$ re-condenses onto olivine, pyroxene, and quartz. The exchange of material between the quartz, olivine, and pyroxene of the annealed pristine dust mixture on the one hand, and of the components forsterite and enstatite of the equilibrium mixture on the other hand, is nearly a one-way process. For a first-order approach to the problem it suffices, then, to neglect the re-condensation of evaporated material onto quartz, olivine, and pyroxene. The exchange of material between the annealed pristine dust components and the equilibrium dust components is schematically represented in Fig. 4.

If in the protoplanetary accretion disc mixing processes intermingle forsterite and enstatite from the inner disc region with pristine dust material from the parent molecular cloud, the gas-phase abundance of $\mathrm{SiO}$ and $\mathrm{Mg}$ atoms in the region where the pristine dust starts to evaporate are essentially determined by the equilibrium between evaporation and recondensation of forsterite and enstatite, while the gas-phase abundance of $\mathrm{Fe}$ atoms is determined by the equilibrium with solid iron. The presence of the equilibrium components acts as a buffer for the partial pressures of their evaporation products $\mathrm{SiO}, \mathrm{Mg}$, and $\mathrm{Fe}$ (forsterite-enstatite buffer). Their abundances, however, do not completely correspond to a chemical equilibrium state, because (i) there occurs a diffusive turbulent transport of gas-phase species from regions of higher to regions of lower concentration within the protoplanetary disc and (ii) there occurs a mass-transport through the gas-phase from the annealed non-equilibrium species quartz, olivine, and pyroxene to the equilibrium species forsterite and enstatite.

Olivine is known to evaporate congruently, i.e. the evaporation residue has the same composition as the starting material (Nagahara et al. 1994). The laboratory experiments of Nagahara \& Ozawa $(1994,1996)$ and Tsuchiyama et al. (1999) on olivine evaporation have shown that at pressures above $1 \times$ $10^{-6}$ bar the evaporation of olivine in the presence of a hot hydrogen gas is determined by a chemical reaction of the type (6). The alternative pure vacuum evaporation reaction

$$
\begin{array}{r}
\mathrm{Mg}_{2 x} \mathrm{Fe}_{2(1-x)} \mathrm{SiO}_{4} \longrightarrow \\
2 x \mathrm{Mg}+2(1-x) \mathrm{Fe}+\mathrm{SiO}+\frac{3}{2} \mathrm{O}_{2}
\end{array}
$$

studied by Hashimoto (1990) governs the evaporation process at $\mathrm{H}_{2}$ pressures below $1 \times 10^{-6}$ bar. Since pressures in the evaporation region in the protoplanetary disc are higher, the chemical reaction (6) is the relevant process for olivine evaporation in a protoplanetary disc.

The evaporation behaviour of pyroxene is more complex because this material evaporates incongruently. Detailed experimental studies of enstatite evaporation in vacuum and hot $\mathrm{H}_{2}$ gas have been performed by Tachibana et al. (2002) who found that enstatite develops a forsterite residue layer during evaporation and that enstatite evaporation probably is controlled by diffusion of $\mathrm{O}$ or $\mathrm{Si}$ in the forsterite boundary layer. They found the steady-state evaporation of enstatite in vacuum and $\mathrm{H}_{2}$ gas to proceed at the evaporation rate of the forsterite residue layer.

\subsection{Equations for the pristine dust components}

For the quartz-, olivine-, and pyroxene-type dust components of interstellar origin two processes are important for the change of their structure and abundance:

1.) At about $800 \mathrm{~K}$ (or $950 \mathrm{~K}$, depending on the adopted experimental data, see Paper I), annealing converts the amorphous lattice structure of the grains from the interstellar medium into a crystalline lattice structure. The consequence of this is that in the warm inner region of the protostellar disc, where decomposition/evaporation processes start to operate, one has to consider the evaporation of crystalline material with its rather well defined properties and not that of amorphous material, the properties of which usually are not well known.

The degree of crystallinity $\xi$ of olivine- and pyroxene-type dust is calculated by the set of equations given in Paper I. 
2.) At a much higher temperature the annealed interstellar quartz, olivine, and pyroxene grains start to evaporate by reaction with $\mathrm{H}_{2}$, which is just a reduction. Here we can apply the approximation that material which evaporated from these grains is completely lost because it precipitates on the more stable dust components forsterite and enstatite.

The decomposition of quartz, olivine, and pyroxene can be treated in the same way as we treated the oxidation of carbon grains in Paper I. We only have to replace expression (17) in Paper I by

$\frac{\mathrm{d} a}{\mathrm{~d} t}=-V_{0} \alpha v_{\mathrm{th}, \mathrm{SiO}} \frac{p_{\mathrm{SiO}, \mathrm{eq}}}{k T}$.

$V_{0}, \alpha$, and $p_{\mathrm{SiO}, \text { eq }}$ are the volume of the nominal molecule in the bulk condensate, the evaporation (sticking) coefficient, and the partial vapour pressure of $\mathrm{SiO}$ in chemical equilibrium with quartz. $v_{\mathrm{th}, \mathrm{SiO}}$ is the root-mean-square velocity of $\mathrm{SiO}$ molecules in the gas-phase

$v_{\text {th }}=\sqrt{\frac{k T}{A m_{\mathrm{AMU}}}}$,

where $A$ is the atomic weight of the growth species (in the present case that of $\mathrm{SiO}$ ), and $m_{\mathrm{AMU}}$ is the atomic mass unit. $\mathrm{d} a / \mathrm{d} t$ may depend on the grain size, for instance by a sizedependent vapour pressure, but we shall assume in our calculations that $\mathrm{d} a / \mathrm{d} t$ does not depend on grain size $a_{i}$. Similar equations hold for olivine and pyroxene. The quantities $V_{0}$ and $\alpha$ for the different dust species are given in Table 2 .

The boundary conditions for the concentration of grains within a given size interval are given by the analogue of Eq. (22) of Paper I, where $f(a)$ in that equation now denotes the interstellar size distributions of the olivine-, pyroxene-, and quartz-type ISM grains. In the normalization condition (21) of Paper I one has to use for $\epsilon$ the Si abundance and for $f$ the fraction of the Si bound in the olivine-, pyroxene- and quartz-type dust components, given in Table 1 .

The evaporation of the olivine, pyroxene, and quartz dust adds $\mathrm{SiO}$ molecules and $\mathrm{Mg}$ and $\mathrm{Fe}$ atoms to the gas-phase. The rate term (5) in Eq. (4) corresponding to the gain of $\mathrm{SiO}$ molecules by evaporation of olivine $\left(\mathrm{Mg}_{2 x} \mathrm{Fe}_{2(1-x)} \mathrm{SiO}_{4}\right)$, pyroxene $\left(\mathrm{Mg}_{x} \mathrm{Fe}_{1-x} \mathrm{SiO}_{3}\right)$ and quartz $\left(\mathrm{SiO}_{2}\right)$ dust is

$$
\begin{aligned}
\frac{R_{m}}{N_{\mathrm{H}}}=\sum_{i} 4 & \pi a_{i}^{2}\left[-\frac{1}{V_{\mathrm{ol}, 0}} \frac{\mathrm{d} a_{\mathrm{ol}, i}}{\mathrm{~d} t} c_{\mathrm{ol}, i}-\frac{1}{V_{\mathrm{py}, 0}} \frac{\mathrm{d} a_{\mathrm{py}, i}}{\mathrm{~d} t} c_{\mathrm{py}, i}\right. \\
& \left.-\frac{1}{V_{\mathrm{qu}, 0}} \frac{\mathrm{d} a_{\mathrm{qu}, i}}{\mathrm{~d} t} c_{\mathrm{qu}, i}\right],
\end{aligned}
$$

where the index $m$ here corresponds to $\mathrm{SiO}$. The quantities $\mathrm{d} a / \mathrm{d} t$ are given by Eq. (10) for each of the three materials. The corresponding expression for the gain of $\mathrm{Mg}$ by evaporation of olivine and pyroxene is

$$
\begin{array}{r}
\frac{R_{\mathrm{Mg}}}{N_{\mathrm{H}}}=\sum_{i} 4 \pi a_{i}^{2}\left[-\frac{1}{V_{\mathrm{ol}, 0}} \frac{\mathrm{d} a_{\mathrm{ol}, i}}{\mathrm{~d} t} 2 x_{\mathrm{ol}} c_{\mathrm{ol}, i}\right. \\
\left.-\frac{1}{V_{\mathrm{py}, 0}} \frac{\mathrm{d} a_{\mathrm{py}, i}}{\mathrm{~d} t} x_{\mathrm{py}} c_{\mathrm{py}, i}\right]
\end{array}
$$

and that for $\mathrm{Fe}$ is

$$
\begin{gathered}
\frac{R_{\mathrm{Fe}}}{N_{\mathrm{H}}}=\sum_{i} 4 \pi a_{i}^{2}\left[-\frac{1}{V_{\mathrm{ol}, 0}} \frac{\mathrm{d} a_{\mathrm{ol}, i}}{\mathrm{~d} t} 2\left(1-x_{\mathrm{ol}}\right) c_{\mathrm{ol}, i}\right. \\
\left.-\frac{1}{V_{\mathrm{py}, 0}} \frac{\mathrm{d} a_{\mathrm{py}, i}}{\mathrm{~d} t}\left(1-x_{\mathrm{py}}\right) c_{\mathrm{py}, i}\right],
\end{gathered}
$$

where $x_{\mathrm{ol}}$ and $x_{\mathrm{py}}$ are the mole fractions of fayalite and ferrosilite in olivine and pyroxene, respectively.

The volume $V_{0}$ in the above equations is given by Eq. (3). Since olivine with composition $\mathrm{Mg}_{2 x} \mathrm{Fe}_{2(1-x)} \mathrm{SiO}_{4}$ forms an ideal solution series between the end members forsterite $\mathrm{Mg}_{2} \mathrm{SiO}_{4}$ and fayalite $\mathrm{Fe}_{2} \mathrm{SiO}_{4}$ we can calculate the density of olivine with mixing ratio $x$ as the linear superposition of the densities of forsterite and fayalite. Using an obvious notation, we have

$\frac{A}{\rho_{\mathrm{sol}}}=\frac{x A_{\mathrm{fo}}+(1-x) A_{\mathrm{fa}}}{x \rho_{\mathrm{fo}}+(1-x) \rho_{\mathrm{fa}}}$.

Similarly we calculate $A / \rho_{\text {sol }}$ for pyroxene. The data and the results for the mixing ratio $x=0.7$ of the P94 dust mixture are given in Table 2 . The values adopted for the sticking coefficient $\alpha$ are given in Table 2 .

\subsection{Cation diffusion}

In olivine and pyroxene the $\mathrm{Mg}^{2+}$ and $\mathrm{Fe}^{2+}$ cations may be inhomogeneously distributed within the dust grains as they enter the accretion disc. At sufficiently high temperature the spatial inhomogeneities are erased, however, by solid diffusion (Gail \& Sedlmayr 1999; Gail 2001). The characteristic timescale for diffusion across a grain of size $a$ is

$t_{\text {diff }}=\frac{a^{2}}{D}$

where $D$ is the diffusion coefficient. The coefficient of cation interdiffusion in olivine is rather well studied by laboratory measurements. The most recent determination is

$D=2 \times 10^{-2} \exp \{-27180 / T-6.91 x\}\left[\mathrm{cm}^{2} \mathrm{~s}^{-1}\right]$

where $x$ is the mole fraction of forsterite in olivine (Chakraborty 1997). The cation interdiffusion in pyroxene is much less well known; especially the dependence on composition is not known. A recent determination for orthopyroxene with $x=0.88$ is (Schwandt et al. 1998)

$D=1.1 \exp \{-43300 / T\}\left[\mathrm{cm}^{2} \mathrm{~s}^{-1}\right]$.

Figure 5 shows the timescales for cation interdiffusion and for radial or vertical mixing as a function of the midplane temperature in a protoplanetary accretion disc model. Homogenization of cation concentration variations within dust grains occurs at about $700 \mathrm{~K}$ for olivine and at about $900 \mathrm{~K}$ for orthopyroxene grains. These temperatures are well below the stability limits of both silicates. If such grains enter the evaporation zone of silicate grains, all internal spatial inhomogeneities with respect to the cation concentrations are erased. It suffices to consider evaporation of grains which are homogeneous with respect to $\mathrm{Mg}^{2+}$ and $\mathrm{Fe}^{2+}$ cation concentrations. 


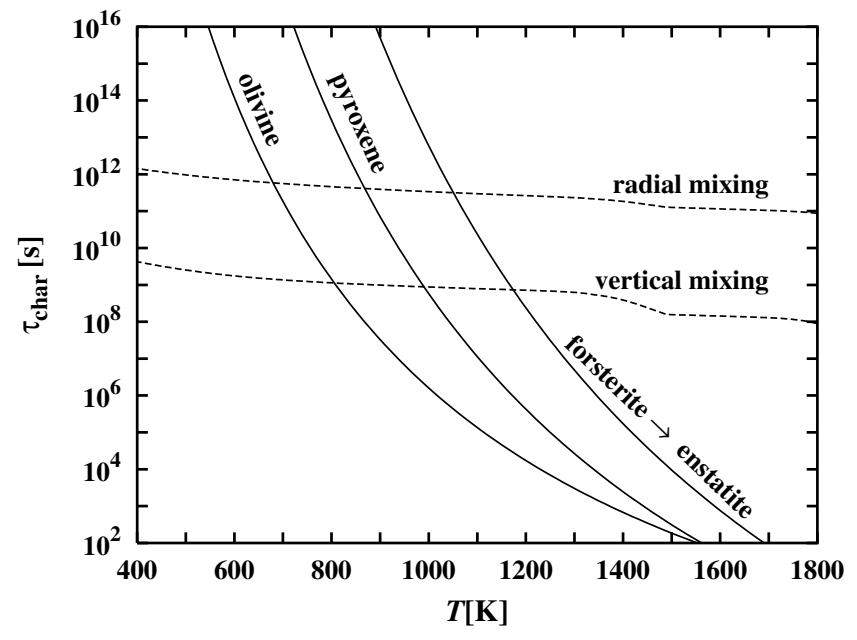

Fig. 5. Temperature variation of the diffusion timescales for $\mathrm{Mg}^{2+}$, $\mathrm{Fe}^{2+}$ cation interdiffusion in olivine and orthopyroxene grains of $0.1 \mu \mathrm{m}$ size with a mole fraction of $\approx 10 \%$ of the iron-bearing end members of the solution series. Also shown is the characteristic timescale for the conversion of a $0.1 \mu \mathrm{m}$ forsterite grain into an enstatite grain in Si-rich vapour. The dashed lines show the variation of the timescales for radial and vertical mixing in a protoplanetary disc with midplane temperature for a disc model with $\dot{M}=10^{-7} M_{\odot} \mathrm{yr}^{-1}$.

\subsection{Equations for the iron component}

\subsubsection{Equations for the size spectrum}

The problem of the iron grains is somewhat more complicated. In the P94 mixture, that part of the iron that is not bound in silicates is present either as troilite (FeS) or as free iron grains. At moderately elevated temperatures activation energy barriers preventing the free iron at low temperatures to be converted into $\mathrm{FeS}$ can be surmounted, and the iron grains react with $\mathrm{H}_{2} \mathrm{~S}$ from the gas-phase to form also FeS. At an even higher temperature of about $700 \mathrm{~K}$ the $\mathrm{FeS}$ is reduced by hydrogen into metallic iron. The details of this process have been studied in the laboratory by Lauretta et al. $(1996,1998)$ and Tachibana \& Tsuchiyama (1998). In the present model calculation we do not consider the conversion of Fe into FeS and back into Fe. In order to hold the number of dust species, i.e. the number of diffusion equations to be considered in the model calculation, at a minimum, we concentrate completely on the processes of conversion of the silicates into the equilibrium mixture which operate at temperatures where no $\mathrm{FeS}$ exists. For this reason we only consider solid iron in our calculations.

The change of the radius of iron grains, Eq. (2), is

$\frac{\mathrm{d} a}{\mathrm{~d} t}=V_{\mathrm{ir}, 0} \alpha_{\mathrm{ir}} v_{\mathrm{th}, \mathrm{Fe}}\left(N_{\mathrm{H}} c_{\mathrm{Fe}}-\frac{p_{\mathrm{Fe}, \mathrm{eq}}}{k T}\right)$.

$V_{\mathrm{ir}, 0}, \alpha_{\mathrm{ir}}, v_{\mathrm{th}, \mathrm{Fe}}$, and $p_{\mathrm{Fe}, \mathrm{eq}}$ are the volume that $\mathrm{Fe}$ atoms occupy in solid iron, the sticking coefficient of iron, the root-meansquare velocity of $\mathrm{Fe}$ atoms in the gas-phase, and the vapour pressure of Fe atoms over solid iron, respectively. $c_{\mathrm{Fe}}$ is the gasphase concentration of $\mathrm{Fe}$ atoms and $N_{\mathrm{H}}$ the particle density of $\mathrm{H}$ nuclei. The sticking coefficient $\alpha_{\text {ir }}$ of iron is discussed e.g. in Gail \& Sedlmayr (1999), Ferrarotti \& Gail (2001) and Gail (2003). The adopted values for $V_{\mathrm{ir}, 0}$ and $\alpha_{\mathrm{ir}}$ also are given in Table 2.
The boundary conditions for the concentration of iron grains within a given size interval are given by the analogue of Eq. (22) of Paper I where $f(a)$ in that equation now denotes the interstellar size distributions of the iron grains. Note that, at the outer boundary, part of the iron is bound in olivine- and pyroxene-type dust. The normalization condition Eq. (21) of Paper I in case of the the size distribution $f(a)$ of iron grains then reads as follows

$$
\begin{aligned}
& \int_{0}^{\infty} \frac{4 \pi}{3} a^{3} f(a)= \\
& V_{\mathrm{ir}, 0}\left[2\left(1-x_{\mathrm{ol}}\right) f_{\mathrm{ol}, \mathrm{ISM}}+\left(1-x_{\mathrm{py}}\right) f_{\mathrm{py}, \mathrm{ISM}}\right] \epsilon_{\mathrm{Si}} .
\end{aligned}
$$

Here $f_{\mathrm{ol}, \mathrm{ISM}}$ and $f_{\mathrm{py}, \mathrm{ISM}}$ denote the degree of condensation of $\mathrm{Si}$ into the pristine olivine- and pyroxene-type grains, respectively. For the P94 mixture they are given in Table 1. $x_{\mathrm{ol}}$ and $x_{\mathrm{py}}$ are the initial mole fractions of forsterite- and enstatite-type material in the olivine- and pyroxene-type solid solutions, respectively. For the P94 dust mixture they are given in Table 1, too. The quantity in brackets is just the fractional abundance of $\mathrm{Fe}$ in the silicate grains.

The contribution of iron evaporation and growth to the rate term (5) in Eq. (4) for the evolution of the concentration of Fe atoms in the gas-phase is

$\frac{R_{\mathrm{Fe}}}{N_{\mathrm{H}}}=\sum_{i} 4 \pi a_{i}^{2} c_{\mathrm{ir}, i}\left(-\frac{1}{V_{i r, 0}} \frac{\mathrm{d} a_{i r, i}}{\mathrm{~d} t}\right)$,

where $\mathrm{d} a / \mathrm{d} t$ is given by Eq. (19).

\subsubsection{Equation for the free iron abundance}

If one adds the advection-diffusion-reaction equations for iron grains and for the iron in the gas-phase, one obtains an equation for the total concentration of the iron not bound in silicates. As long as the size of the iron grains is less than $\approx 1 \mathrm{~mm}$ the iron grains are dynamically coupled to the gas (cf. Fig. 3 of Weidenschilling \& Cuzzi 1993) such that their transport coefficients $D$ and $v_{\mathrm{r}}$ are the same as that for the gas-phase species. The resulting equation is

$\frac{\partial \epsilon_{\mathrm{Fe}}^{*}}{\partial t}+v_{\mathrm{r}} \frac{\partial \epsilon_{\mathrm{Fe}}^{*}}{\partial r}=\frac{1}{n r} \frac{\partial}{\partial r} r n D \frac{\partial \epsilon_{\mathrm{Fe}}^{*}}{\partial r}+\frac{R_{\mathrm{Fe}^{*}}}{N_{\mathrm{H}}}$

where $R_{\mathrm{Fe}^{*}} / N_{\mathrm{H}}$ is given by Eq. (14). The quantity $\epsilon_{\mathrm{Fe}}^{*}$ is the element abundance of Fe (relative to hydrogen nuclei) not bound in silicates. Terms corresponding to evaporation and condensation of solid iron cancel from this equation; only the production term resulting from evaporation of olivine and pyroxene remains.

The boundary conditions required for solving Eq. (22) obviously are as follows: at the inner boundary $r_{\mathrm{i}}$ of the disc model we have

$\epsilon_{\mathrm{Fe}}^{*}\left(r_{\mathrm{i}}\right)=\epsilon_{\mathrm{Fe}}$,

where $\epsilon_{\mathrm{Fe}}$ is the total element abundance of Fe relative to $\mathrm{H} \mathrm{nu}-$ clei in the matter from the parent molecular cloud. At the outer boundary $r_{\mathrm{o}}$ of the disc model we have

$$
\begin{aligned}
\epsilon_{\mathrm{Fe}}^{*}\left(r_{\mathrm{o}}\right)= & \epsilon_{\mathrm{Fe}} \\
& -\left(2\left(1-x_{\mathrm{ol}}\right) f_{\mathrm{ol}, \mathrm{ISM}}+\left(1-x_{\mathrm{py}}\right) f_{\mathrm{py}, \mathrm{ISM}}\right) \epsilon_{\mathrm{Si}}
\end{aligned}
$$


which is the total $\mathrm{Fe}$ abundance of the interstellar matter from which the disc is formed, reduced by the fraction of the iron bound in silicates.

In Duschl et al. (1996) it is shown that the timescale for evaporation or condensation is much smaller than the characteristic timescale for the change of the pressure and temperature of a comoving gas element in the disc. The difference between the gas-phase partial pressure of free $\mathrm{Fe}$ atoms and the vapour pressure of iron is very small in this case. If we neglect this small difference, the degree of condensation of $\mathrm{Fe}$ into iron grains is given by

$f_{\text {ir }}=\frac{\epsilon_{\mathrm{Fe}}^{*}}{\epsilon_{\mathrm{Fe}}}-\frac{p_{\mathrm{Fe}, \mathrm{eq}}}{\epsilon_{\mathrm{Fe}} N_{\mathrm{H}} k T}$.

This quantity, which is important for the construction of disc models, can already be obtained from the solution of Eq. (22).

\subsubsection{Equation for the gas-phase iron abundance}

The equation for the concentration of iron atoms in the gasphase is according to Eq. (4)

$$
\frac{\partial c_{\mathrm{Fe}}}{\partial t}+v_{\mathrm{r}} \frac{\partial c_{\mathrm{Fe}}}{\partial r}=\frac{1}{n r} \frac{\partial}{\partial r} r n D \frac{\partial c_{\mathrm{Fe}}}{\partial r}+\frac{R_{\mathrm{Fe}}}{N_{\mathrm{H}}} .
$$

The source term $R_{\mathrm{Fe}} / N_{\mathrm{H}}$ consists of several contributions:

- The gain terms Eq. (14) corresponding to evaporation of the annealed pristine olivine and pyroxene grains and

- the gain and loss terms Eq. (21) from evaporation and condensation of solid iron.

If, as we shall always assume, the evaporation and condensation rates do not depend on the grain size (i.e. we neglect the increase of vapour pressure with decreasing radius of curvature of the grain surface) then we may take the rates per unit surface out of the summation over the size spectrum. We introduce for each dust species $j$ the total surface area of grains per $\mathrm{H}$ nucleus by

$\mathcal{A}_{j}=\sum_{i} 4 \pi a_{i}^{2} c_{j, i}$.

Then we have

$$
\begin{aligned}
\frac{R_{\mathrm{Fe}}}{N_{\mathrm{H}}}= & -\alpha_{\mathrm{ir}} v_{\mathrm{th}, \mathrm{Fe}}\left(N_{\mathrm{H}} c_{\mathrm{Fe}}-\frac{p_{\mathrm{Fe}, \mathrm{eq}}}{k T}\right) \mathcal{A}_{\mathrm{Fe}} \\
& +2\left(1-x_{\mathrm{ol}}\right) \alpha_{\mathrm{ol}} v_{\mathrm{th}, \mathrm{SiO}} \frac{p_{\mathrm{SiO}, \mathrm{eq}}^{\mathrm{ol}}}{k T} \mathcal{A}_{\mathrm{ol}} \\
& +\left(1-x_{\mathrm{py}}\right) \alpha_{\mathrm{py}} v_{\mathrm{th}, \mathrm{SiO}} \frac{p_{\mathrm{SiO}, \mathrm{eq}}^{\mathrm{py}}}{k T} \mathcal{A}_{\mathrm{py}} .
\end{aligned}
$$

The differential Eq. (26) with this source term (28) determines the gas-phase abundance of free Fe atoms.

The source terms corresponding to the evaporation of olivine and pyroxene are important only over a limited temperature region since $p_{\mathrm{SiO} \text {,eq }}$ strongly increases with temperature (approximately exponentially) and $\mathcal{A}_{\mathrm{ol}}$ and $\mathcal{A}_{\mathrm{py}}$ rapidly go to zero if the grains evaporate.

\subsubsection{Deviation from equilibrium}

First we consider the temperature region where evaporation of olivine and pyroxene is inefficient or both species are absent. In that case the source and sink terms due to iron evaporation and condensation, respectively, on the r.h.s. of Eq. (26) have to compensate the drift and diffusion terms on the 1.h.s. of the equation.

Since the characteristic timescale for diffusion and inwards transport of matter are rather long there is ample time for establishing a near-equilibrium state in which the gas-phase partial pressure of $\mathrm{Fe}$ atoms nearly equals the vapour pressure of iron

$c_{\mathrm{Fe}, \mathrm{eq}} N_{\mathrm{H}} \approx \frac{p_{\mathrm{Fe}, \mathrm{eq}}}{k T}$

such that the small difference between $c_{\mathrm{Fe}, \mathrm{eq}} N_{\mathrm{H}}$ and $p_{\mathrm{Fe}, \mathrm{eq}} / k T$ results in a growth/evaporation time scale of the same order of magnitude as the shorter one of the drift or diffusion timescales.

To estimate the order of magnitude to be expected for the deviation between the gas-phase abundance $c_{\mathrm{Fe}, \mathrm{eq}} N_{\mathrm{H}}$ of $\mathrm{Fe}$ atoms and their chemical equilibrium abundance $p_{\mathrm{Fe}, \text { eq }} / k T$ we compare the first term on the right hand side of Eq. (28) with the drift term. The diffusion term is of the same order of magnitude as the drift term since for $\alpha$-discs $D=\frac{2}{3} r v_{\mathrm{r}}$ (see Paper I).

As an order-of-magnitude approximation we obtain for the ratio of the growth/evaporation term to the drift term

$\frac{\alpha v_{\mathrm{th}} N_{\mathrm{H}} c_{\mathrm{Fe}} \mathcal{A} r}{v_{\mathrm{r}} c_{\mathrm{Fe}}}\left(1-\frac{1}{S}\right)$

where $S$ is the ratio

$S=\frac{c_{\mathrm{Fe}}}{c_{\mathrm{Fe}, \mathrm{eq}}}$

and

$c_{\mathrm{Fe}, \mathrm{eq}}=\frac{p_{\mathrm{Fe}, \mathrm{eq}}}{N_{\mathrm{H}} k T}$.

Assuming for an order-of-magnitude estimate that all grains have the same radius $a$ we have $\mathcal{A}=4 \pi a^{2} n_{\mathrm{gr}} / N_{\mathrm{H}}$, where $n_{\mathrm{gr}}$ is the number density of grains. At about $1 \mathrm{AU}$ the total density of $\mathrm{H}$ nuclei is about $10^{15} \mathrm{~cm}^{-3}$ and $n_{\mathrm{gr}}$ then is of the order of $100 \mathrm{~cm}^{-3}$.

Then we have to estimate the order of magnitude of

$\frac{\alpha v_{\mathrm{th}} 4 \pi a^{2} n_{\mathrm{gr}} r}{v_{\mathrm{r}}}\left(1-\frac{1}{S}\right)$.

With typical values of $v_{\mathrm{th}}=10^{4} \mathrm{~cm} \mathrm{~s}^{-1}, a=10^{-5} \mathrm{~cm}, n_{\mathrm{gr}}=$ $10^{2} \mathrm{~cm}^{-3}, r=10^{13} \mathrm{~cm}$ and $v_{\mathrm{r}}=10 \mathrm{~cm} \mathrm{~s}^{-1}$ we obtain

$10^{8}\left(1-\frac{1}{S}\right)$.

Since this has to be of order unity this means that $S$ deviates from unity only by about $10^{-8}$, i.e., with a high degree of accuracy we have $c_{\mathrm{Fe}}=c_{\mathrm{Fe} \text {,eq }}$.

Next consider the region where olivine and pyroxene evaporation is important. In this region we also have to consider the gain terms from this process. We compare the order of magnitude of the growth/evaporation term of iron grains to that of the 
gain term due to olivine evaporation. Their ratio is of the order of magnitude

$$
\frac{\alpha_{\mathrm{ir}} p_{\mathrm{Fe}, \mathrm{eq}} \mathcal{A}_{\mathrm{Fe}}}{\alpha_{\mathrm{ol}} p_{\mathrm{SiO}, \mathrm{eq}} \mathcal{A}_{\mathrm{ol}}}\left(1-\frac{1}{S}\right) .
$$

If the olivine is not yet nearly completely evaporated, the quantities $\mathcal{A}_{\mathrm{ol}}$ and $\mathcal{A}_{\mathrm{Fe}}$ are of comparable order of magnitude. Since $p_{\mathrm{Fe}, \text { eq }}$ exceeds $p_{\mathrm{SiO} \text {,eq }}$ by about two orders of magnitude in the temperature regime $1000 \ldots 1100 \mathrm{~K}$ (cf. Fig. 3) and $\alpha_{\text {ir }} \approx 10 \alpha_{\text {ol }}$ (cf. Table 2), the ratio of the two terms is

$10^{3}\left(1-\frac{1}{S}\right)$

Then the source terms from silicate evaporation are much bigger than the transport and diffusion terms from the 1.h.s. of Eq. (26) and they have to compensate the growth/evaporation term from the r.h.s. This requires that the deviation of $S$ from unity is very small, of the order of $10^{-3}$, i.e., also in the present case we have $c_{\mathrm{Fe}}=c_{\mathrm{Fe} \text {,eq }}$ with a high degree of accuracy, but the deviations are not as small as in the preceding case.

The fact that $c_{\mathrm{Fe}}$ is very close to $c_{\mathrm{Fe} \text {,eq }}$ would allow us to replace $c_{\mathrm{Fe}}$ on the left hand side of Eq. (26) and in the diffusion term by $c_{\mathrm{Fe} \text {,eq }}$. Since $c_{\mathrm{Fe} \text {,eq }}$ for a given disc model is a known quantity, Eq. (26) reduces to a simple algebraic equation from which we could calculate the difference $c_{\mathrm{Fe}}-c_{\mathrm{Fe} \text {,eq }}$, if we know the quantities $\mathcal{A}_{j}$. Numerical test calculations showed that, indeed, the results for $c_{\mathrm{Fe}}-c_{\mathrm{Fe} \text {,eq }}$ calculated in this way are the same as that obtained from a solution of Eq. (26), but they also showed that the simpler approach has no advantages over the direct solution of Eq. (26) because the numerical differentiation of $c_{\mathrm{Fe}, \mathrm{eq}}$ is sensitive to numerical noise from the iterations required for the temperature calculation.

These estimates all refer to radial transport processes. If vertical transport processes were considered, the deviations from equilibrium would become bigger because of shorter vertical transport timescales. Within the frame of the one-zone model for which we do our model calculations, vertical transport in the accretion disc cannot be treated and so we do not consider this case, but one should bear in mind that the real deviations from equilibrium may not be as extremely small as found in the above estimates.

\subsection{Equations for the solar nebula condensation products forsterite and enstatite}

\subsubsection{Equations for the size spectrum}

For the dust components of the $\mathrm{Mg}$-Si-complex resulting from evaporation and re-condensation processes we have to consider the species forsterite and enstatite. In Duschl et al. (1996) we have shown that these dust components grow and evaporate in the warm inner parts of the protostellar disc under nearequilibrium conditions. At the vaporization limit all forsterite and enstatite grains are destroyed. The existence of such grains at lower temperatures, thus, requires that seed nuclei for the growth of forsterite and enstatite are formed somewhere in the disc. Direct nucleation from the gas-phase is not possible because the nominal molecules of both minerals do not exist in the gas-phase. The forsterite and enstatite grains instead have to grow on top of some different kind of seed nuclei. In the chemical mixture of the protoplanetary disc there exist many mineral compounds which are stable up to much higher temperatures than forsterite and enstatite and are possible candidates for serving as growth centres for the magnesium silicates, for instance Ca-Al-compounds, Ti compounds like perovskite, zirconium oxide ..., just to mention a few. Figure 2 shows the stability limits of some of these compounds. The precise nature of the seed nuclei does not play any role, as long as such seed nuclei exist. We assume in our model calculation that the equilibrium condensates forsterite and enstatite grow on some kind of pre-formed seed nuclei, the nature of which needs not be specified.

This behaviour can be simulated in the numerical solution of the advection-diffusion-reaction Eqs. (1) by omitting the loss-term

$$
-\frac{c_{j, i}}{\Delta a_{i}}\left|\frac{\mathrm{d} a_{j, i}}{\mathrm{~d} t}\right|
$$

from the equation for the smallest grain size in the case $\mathrm{d} a_{i, j} / \mathrm{d} t<0$. These smallest grains then formally act as the growth centres, onto which material grows if $\mathrm{d} a_{i, j} / \mathrm{d} t>0$, but which are never destroyed. The concentration of such grains (seed particles) has to be specified in this approach as an inner boundary condition for the corresponding concentration $c_{j, i}$.

The change of the radius of the grains of forsterite or enstatite, Eq. (2), is given by

$\frac{\mathrm{d} a}{\mathrm{~d} t}=V_{0} \alpha v_{\mathrm{th}, \mathrm{SiO}}\left(N_{\mathrm{H}} c_{\mathrm{SiO}}-\frac{p_{\mathrm{SiO}, \mathrm{eq}}}{k T}\right)$.

The meaning of the quantities entering into this expression is analogous to that in the above cases. The values of the sticking coefficients $\alpha$ of forsterite and enstatite are discussed e.g. in Gail \& Sedlmayr (1999), in Ferrarotti \& Gail (2001) and Gail (2003). The adopted values for $V_{0}$ and $\alpha$ are also given in Table 2.

The boundary conditions required for solving Eqs. (1) for the particle size spectrum $c_{j, i}$ at the inner and outer disc boundaries are as follows: At the outer boundary $r_{\mathrm{o}}$ of the disc model we simply have

$c_{j, i}\left(r_{\mathrm{o}}\right)=0$,

since the enstatite and forsterite grains are not members of the pristine dust mixture. At the inner boundary $r_{\mathrm{i}}$ of the disc model the forsterite and enstatite grains are completely evaporated, i.e. we have

$c_{j, i}\left(r_{\mathrm{i}}\right)=0$,

except for the smallest grain size $a_{i}$ for which we have to prescribe

$c_{j, i}\left(r_{\mathrm{i}}\right)=c_{\text {grains, } j}$.

$j$ denotes the dust species, forsterite and enstatite in our case, and $c_{\text {grains, } j}$ is the total number of grains per hydrogen nuclei of that dust species. This concentration of seed nuclei must be guessed in some way. 


\subsubsection{Equations for the abundance of $\mathrm{Si}$ and $\mathrm{Mg}$ not in pristine dust}

If one adds the advection-diffusion-reaction equations for forsterite and enstatite grains and for the $\mathrm{SiO}$ molecules in the gas-phase, one obtains an equation for the total concentration of the Si not bound in the pristine silicate dust components of olivine-, pyroxene- and quartz-type composition. The resulting equation, analogous to the case of iron, is

$\frac{\partial \epsilon_{\mathrm{Si}}^{*}}{\partial t}+v_{\mathrm{r}} \frac{\partial \epsilon_{\mathrm{Si}}^{*}}{\partial r}=\frac{1}{n r} \frac{\partial}{\partial r} r n D \frac{\partial \epsilon_{\mathrm{Si}}^{*}}{\partial r}+\frac{R_{\mathrm{Si}}}{N_{\mathrm{H}}}$,

where $R_{\mathrm{Si}} / N_{\mathrm{H}}$ is given by Eq. (12). Terms corresponding to evaporation and condensation of forsterite and enstatite, again, cancel from this equation, only the production terms resulting from evaporation of olivine, pyroxene and quartz remain. The quantity $\epsilon_{\mathrm{Si}}^{*}$ is the element abundance of Si relative to hydrogen nuclei not bound in the pristine silicates. This silicon either is bound in forsterite and enstatite or is present as molecular species (mainly $\mathrm{SiO}$ ) in the gas-phase.

The boundary conditions required for solving Eq. (36) obviously are as follows: at the inner boundary $r_{\mathrm{i}}$ of the disc model we have

$\epsilon_{\mathrm{Si}}^{*}\left(r_{\mathrm{i}}\right)=\epsilon_{\mathrm{Si}}$,

where $\epsilon_{\mathrm{Si}}$ is the total element abundance of Si relative to $\mathrm{H}$ nuclei in the matter from the parent molecular cloud. At the outer boundary $r_{\mathrm{o}}$ of the disc model we have

$\epsilon_{\mathrm{Si}}^{*}\left(r_{\mathrm{o}}\right)=0$

since all $\mathrm{Si}$ is bound in the pristine silicates.

For the abundance of $\mathrm{Mg}$ not bound in the pristine dust components we obtain in complete analogy

$\frac{\partial \epsilon_{\mathrm{Mg}}^{*}}{\partial t}+v_{\mathrm{r}} \frac{\partial \epsilon_{\mathrm{Mg}}^{*}}{\partial r}=\frac{1}{n r} \frac{\partial}{\partial r} r n D \frac{\partial \epsilon_{\mathrm{Mg}}^{*}}{\partial r}+\frac{R_{\mathrm{Mg}}}{N_{\mathrm{H}}}$,

where $R_{\mathrm{Mg}} / N_{\mathrm{H}}$ is given by Eq. (13). The boundary equations for (39) are

$\epsilon_{\mathrm{Mg}}^{*}\left(r_{\mathrm{i}}\right)=\epsilon_{\mathrm{Mg}}$

at the inner boundary $r_{\mathrm{i}}$ of the disc model and

$\epsilon_{\mathrm{Mg}}^{*}\left(r_{\mathrm{o}}\right)=0$

at the outer boundary $r_{\mathrm{o}}$ of the disc model.

\subsubsection{Equations for the gas-phase abundance of $\mathrm{SiO}$ and $\mathrm{Mg}$}

The equation for the concentration of silicon in the gas-phase is according to Eq. (4)

$$
\frac{\partial c_{\mathrm{Si}}}{\partial t}+v_{\mathrm{r}} \frac{\partial c_{\mathrm{Si}}}{\partial r}=\frac{1}{n r} \frac{\partial}{\partial r} r n D \frac{\partial c_{\mathrm{Si}}}{\partial r}+\frac{R_{\mathrm{SiO}}}{N_{\mathrm{H}}} .
$$

Neglecting the small contribution of Si-bearing molecules different from $\mathrm{SiO}$, the gas-phase $\mathrm{Si}$ concentration is identical with the concentration of $\mathrm{SiO}$ molecules. The source term $R_{\mathrm{Si}} / N_{\mathrm{H}}$ has two contributions: (i) the gain term (12) corresponding to evaporation of the annealed pristine olivine and pyroxene grains; (ii) the gain and loss terms, which describe the contributions of evaporation and condensation of forsterite $\mathrm{Mg}_{2} \mathrm{SiO}_{4}$ and enstatite $\mathrm{MgSiO}_{3}$ to the change of the gas-phase $\mathrm{SiO}$ concentration

$\frac{R_{\mathrm{SiO}}}{N_{\mathrm{H}}}=\sum_{i} 4 \pi a_{i}^{2}\left[-\frac{1}{V_{\mathrm{fo}, 0}} \frac{\mathrm{d} a_{\mathrm{fo}, i}}{\mathrm{~d} t} c_{\mathrm{fo}, i}-\frac{1}{V_{\mathrm{en}, 0}} \frac{\mathrm{d} a_{\mathrm{en}, i}}{\mathrm{~d} t} c_{\mathrm{en}, i}\right]$.

If we assume as in the case of iron evaporation that the evaporation and growth rates do not depend on the grain size we can take the rate terms out of the summation over the grain size spectrum. The rate term in Eq. (42) then is

$$
\begin{aligned}
\frac{R_{\mathrm{SiO}}}{N_{\mathrm{H}}}= & -\alpha_{\mathrm{fo}} v_{\mathrm{th}, \mathrm{SiO}}\left(N_{\mathrm{H}} c_{\mathrm{Si}}-\frac{p_{\mathrm{SiO}, \mathrm{eq}}^{\mathrm{fo}}}{k T}\right) \mathcal{A}_{\mathrm{fo}} \\
& -\alpha_{\mathrm{en}} v_{\mathrm{th}, \mathrm{SiO}}\left(N_{\mathrm{H}} c_{\mathrm{Si}}-\frac{p_{\mathrm{SiO}, \mathrm{eq}}^{\mathrm{en}}}{k T}\right) \mathcal{A}_{\mathrm{en}} \\
& +\alpha_{\mathrm{ol}} v_{\mathrm{th}, \mathrm{SiO}} \frac{p_{\mathrm{SiO}, \mathrm{eq}}^{\mathrm{ol}}}{k T} \mathcal{A}_{\mathrm{ol}} \\
& +\alpha_{\mathrm{py}} v_{\mathrm{th}, \mathrm{SiO}} \frac{p_{\mathrm{SiO}, \mathrm{eq}}^{\mathrm{py}}}{k T} \mathcal{A}_{\mathrm{py}} \\
& +\alpha_{\mathrm{qu}} v_{\mathrm{th}, \mathrm{SiO}} \frac{p_{\mathrm{SiO}, \mathrm{eq}}^{\mathrm{qu}}}{k T} \mathcal{A}_{\mathrm{qu}} .
\end{aligned}
$$

Equation (42) has to be solved with the obvious boundary conditions

$c_{\mathrm{Si}}=\epsilon_{\mathrm{Si}} \quad$ at $r=r_{\mathrm{i}}$

and

$c_{\mathrm{Si}}=0 \quad$ at $r=r_{\mathrm{O}}$.

For the concentration of magnesium one obtains the analogue of Eq. (42) with the source term

$$
\begin{aligned}
\frac{R_{\mathrm{Mg}}}{N_{\mathrm{H}}}= & -2 \alpha_{\mathrm{fo}} v_{\mathrm{th}, \mathrm{SiO}}\left(N_{\mathrm{H}} c_{\mathrm{Mg}}-\frac{p_{\mathrm{Mg}, \mathrm{eq}}^{\mathrm{fo}}}{k T}\right) \mathcal{A}_{\mathrm{fo}} \\
& -\alpha_{\mathrm{en}} v_{\mathrm{th}, \mathrm{SiO}}\left(N_{\mathrm{H}} c_{\mathrm{Mg}}-\frac{p_{\mathrm{Mg}, \mathrm{eq}}^{\mathrm{en}}}{k T}\right) \mathcal{A}_{\mathrm{en}} \\
& +2 x_{\mathrm{ol}} \alpha_{\mathrm{ol}} v_{\mathrm{th}, \mathrm{SiO}} \frac{p_{\mathrm{SiO}, \mathrm{eq}}^{\mathrm{ol}}}{k T} \mathcal{A}_{\mathrm{ol}} \\
& +x_{\mathrm{py}} \alpha_{\mathrm{py}} v_{\mathrm{th}, \mathrm{SiO}} \frac{p_{\mathrm{SiO}, \mathrm{eq}}^{\mathrm{py}}}{k T} \mathcal{A}_{\mathrm{py}} .
\end{aligned}
$$

The boundary conditions in this case are

$c_{\mathrm{Mg}}=\epsilon_{\mathrm{Mg}} \quad$ at $r=r_{\mathrm{i}}$

and

$c_{\mathrm{Mg}}=0$ at $r=r_{\mathrm{o}}$.

The estimates of Sect. 5.4.3 for the order of magnitude of the advection and diffusion term on the one hand and the source and sink terms on the r.h.s of the advection-diffusion-reaction equation on the other hand can also be applied to the present case. They show that the differences between the equilibrium 
Table 3. Parameters used for the calculation of the disc model.

\begin{tabular}{lrrl}
\hline \hline accretion rate & $\dot{M}$ & $10^{-6}, 10^{-7}, 10^{-8}$ & $M_{\odot} \mathrm{yr}^{-1}$ \\
stellar mass & $M_{*}$ & 1 & $M_{\odot}$ \\
effective temperature & $T_{\text {eff }}$ & 4500 & $\mathrm{~K}$ \\
stellar luminosity & $L_{*}$ & 5 & $L_{\odot}$ \\
viscosity parameter & $\alpha$ & $3 \times 10^{-3}$ & \\
molecular cloud temp. & $T_{\text {mol }}$ & 20 & $\mathrm{~K}$ \\
inner disc radius & $r_{\text {in }}$ & 5 & $R_{*}$ \\
outer disc radius & $r_{\text {out }}$ & 100 & $\mathrm{AU}$ \\
\hline
\end{tabular}

gas-phase concentrations of $\mathrm{SiO}$ and $\mathrm{Mg}$ and the actual concentrations defined by the solutions of the advection-diffusionreaction equations ought to be small. The concentration differences, again, can be calculated by replacing $c_{\mathrm{SiO}}$ and $c_{\mathrm{Mg}}$ in the advection and diffusion term of the advection-diffusionreaction equations for $\mathrm{SiO}$ and $\mathrm{Mg}$ by

$c_{\mathrm{SiO}, \mathrm{eq}}=\frac{p_{\mathrm{SiO}, \mathrm{eq}}}{N_{\mathrm{H}} k T}$

and

$c_{\mathrm{Mg}, \mathrm{eq}}=\frac{p_{\mathrm{Mg}, \mathrm{eq}}}{N_{\mathrm{H}} k T}$,

respectively. Since for a given disc model the equilibrium concentrations are known quantities, the concentration differences can be calculated from a simple set of algebraic equations.

\section{Model calculation}

\subsection{Disk model}

The calculation of the conversion of the pristine dust from the molecular cloud into the equilibrium dust mixture in the inner disc region requires us to calculate a self-consistent model for the accretion disc including the transport and mixing processes and the evaporation and re-condensation of the dust components. This determines some important input parameters for the calculation of the dust-related chemical processes, the temperature for instance, which depend themselves on the dust composition in the disc. The calculation follows essentially the methods described in Paper I.

Models are calculated for a protostellar accretion disc around a single star with the set of parameters shown in Table 3. The model is a stationary one-zone Keplerian $\alpha$-disc model. The stellar parameters correspond to a star of one solar mass in a stage of evolution where the star is already visible, i.e. the process of mass infall and star formation is nearly finished and the remaining disc gradually disappears by accretion onto the star (cf. Stahler \& Walter 1993). This is just that phase of the disc evolution that we want to consider. The viscosity parameter $\alpha$ is set to a value which results in a timescale for disc disappearance in time-dependent one-zone model calculations which is of the order of the observed timescale of roughly $10^{6}$ years (Ruden \& Pollack 1991; Paper II). Slightly higher values for $\alpha$ are favoured by others (e.g. Lin \& Papaloizou 1996; Stepinski 1998).

The calculation is done for a mass accretion rate of $\dot{M}=$ $10^{-7} M_{\odot} \mathrm{yr}^{-1}$. This rate is suggested by typical disc masses of $0.1 M_{\odot}$ around solar mass stars and typical disc lifetimes of $10^{6}$ years (e.g. Beckwith \& Sargent 1993; Haisch et al. 2002). This accretion rate is generally thought to be representative for the early stages of the evolution of a protoplanetary disc prior to the onset of planetary formation. For comparison also models with an accretion rate of $10^{-6}$ and $10^{-8} M_{\odot} \mathrm{yr}^{-1}$ are calculated. The former corresponds to an early evolutionary stage where the parent molecular cloud is not yet dissipated and the system is hidden behind a thick dust shell. The latter mass accretion rate probably corresponds to a late stage of the disc evolution where planet formation already is underway. Observationally determined mass accretion rates for young stellar objects of age less than $10^{6} \mathrm{yr}$ seem to support our assumption of a mass accretion rate of $10^{-7} M_{\odot} \mathrm{yr}^{-1}$ for the pre-planetary phase (e.g. Hartmann 2000; Calvet et al. 2000).

\subsection{Opacities}

The opacities of the different dust species are treated by calculating the opacity $\kappa$ entering the disc equations by the linear superposition

$\kappa=\sum_{i} f_{i} \kappa_{i}$

The individual $\kappa_{i}$ are Rosseland means of the opacity of dust species $i$ calculated from Mie theory and a MRN size distribution (Mathis et al. 1977). This clearly is a simplification because Rosseland means are not additive, except in regions where one species dominates the whole extinction or if we deal with grey absorption.

The opacities $\kappa_{i}$ are calculated for the case of $100 \%$ condensation. They are multiplied by the true degree of condensation $f_{i}$ to account for the true abundance of the dust species in the mixture.

For the opacity of the unmodified amorphous silicate dust grains of interstellar origin for both species optical constants of Draine (1985) are used. Also for the carbon dust component the optical constants from the tabulation of Draine have been used. This choice was made in order that the pristine dust component yields opacities that at least are similar to that of interstellar dust.

For the annealed crystalline silicate grains of interstellar origin dust optical constants for pyroxene with a mole fraction of ferrosilite of $x \approx=0.5$ in the solid solution are taken from the database of the Astrophysical Institute of the University of Jena ${ }^{1}$ and for olivine optical constants of a natural specimen from Huffman \& Stapp (1972) and Steyer (1974) are used. These data are the only ones available for materials which may have some similarity in their composition to the annealed interstellar silicates and which cover the extended wavelength region $(0.2 \ldots 300 \mu \mathrm{m})$ necessary for purposes of model calculation.

${ }^{1}$ See: http://www.astro. uni-jena.de/ 
For the disc-made forsterite and enstatite dust grains optical constants for nearly-iron-free silicates are taken from the database of the Astrophysical Institute of the University of Jena; they are supplemented by data from glassy materials in the short-wavelength region.

For the other dust materials the same data are used as in Paper I.

\subsection{Method of model construction}

For each of the dust species we use a grid of 25 grid points $a_{i}$ between $a_{1}=3 \AA$ and $a_{25}=0.25 \mu \mathrm{m}$ for the grain-size spectrum. The initial size distribution at the outer border of the disc is set to the MRN size distribution (Mathis et al. 1977). Different size distributions for interstellar grains have been proposed, e.g. by Weingartner \& Draine (2001), but it is questionable that such a model can be used as initial size distribution at the outer border of the model because the very small grain size component of the model may not survive the passage through the accretion shock. The sizes of grains in the parent molecular clouds of newly formed stars often seem to be bigger than ISM grains due to coagulation. In view of the probably weak forces responsible for coagulation in the cold clouds it seems likely, however, that such grain clusters are disrupted in passing the accretion shock and the ISM size distribution is therefore restored behind the shock.

The disc model is constructed by a fixed-point iteration method. With an initial estimate for the degrees of condensation and crystallinity of the various dust species, a model for the radial disc structure is calculated as described in Paper I. With this model, silicate annealing and carbon dust combustion are calculated by the methods described in Paper I.

Next the evaporation of olivine, pyroxene and quartz is calculated by solving the set of 25 advection-diffusion-reaction Eqs. (1) for each of the species (see Sect. 5.2). This provides the injection rate of $\mathrm{Mg}, \mathrm{Fe}$, and $\mathrm{SiO}$ into the gas-phase.

Next Eqs. (22), (36) and (39) for the abundances of Mg, Fe, and $\mathrm{Si}$ not bound in the pristine dust components are solved. For these abundances the problem of chemical equilibrium between the gas-phase and the solids is solved, which provides molecular abundances in the gas-phase and the different degrees of condensation $f_{j}$ of the solids in chemical equilibrium.

The whole set of calculations is then repeated by calculating a new disc model with the results for the degrees of condensation and the degrees of crystallinity of the various dust species. Then, again, new degrees of condensation and degrees of crystallinity of the various dust species are calculated. The procedure is repeated until relative deviations in the physical quantities between subsequent iteration steps do not change by more then $10^{-7}$. This usually is achieved within about 12 iteration steps.

\subsection{General disc structure}

Figure 6 shows the structure of the stationary disk model for a set of mass-accretion rates of $\dot{M}=10^{-6}, 10^{-7}, 10^{-8} M_{\odot} \mathrm{yr}^{-1}$. The overall disc structure is not significantly changed by the

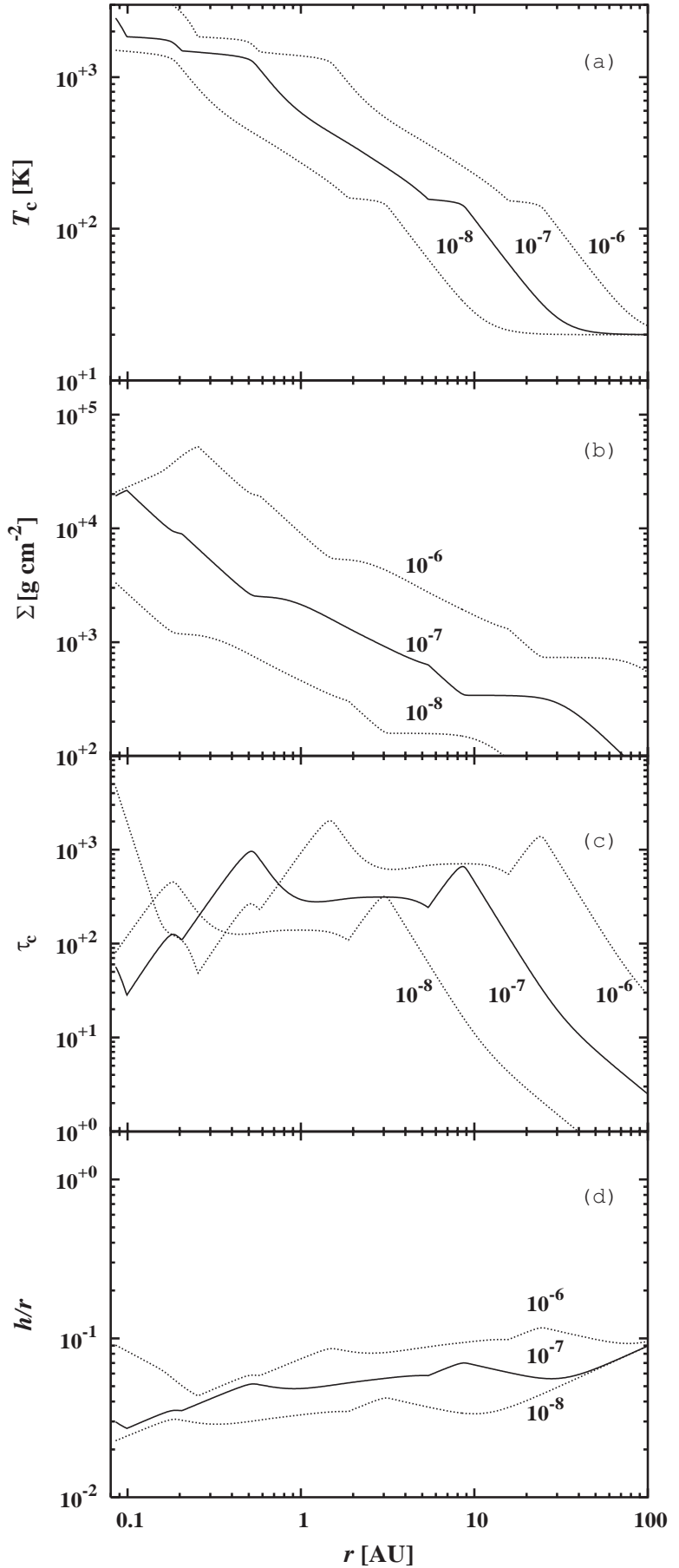

Fig. 6. Model of the radial disc structure for mass accretion rates of $\dot{M}=10^{-6}, 10^{-7}, 10^{-8} M_{\odot} \mathrm{yr}^{-1}$. a) Temperature $T_{\mathrm{c}}$ at the midplane of the disk. b) Surface density $\Sigma$ of the disc. c) Vertical optical depth $\tau_{\mathrm{c}}$ at the midplane. d) Vertical height $h$ of the disk in units of the radius $r$ (aspect ratio).

improved calculation of the dust composition in the disc, as can be seen by comparing Fig. 6 with Fig. 2 of Paper I. The reason is that the total amount of silicate dust does not change much by the present improved model calculation for the dust mixture, but only the distribution of $\mathrm{Si}, \mathrm{Mg}$, and $\mathrm{Fe}$ between the different dust species. This results in only moderate opacity 


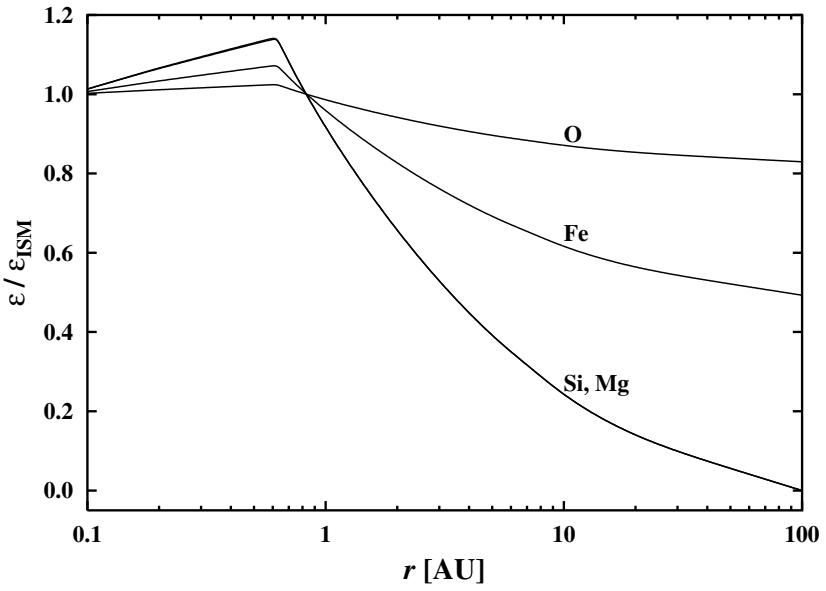

Fig. 7. Abundance of the elements $\mathrm{Fe}, \mathrm{Mg}, \mathrm{Si}$, and $\mathrm{O}$ not bound in the ISM dust components normalized to Solar System abundances. Disc model with accretion rate $\dot{M}=1 \times 10^{-7} M_{\odot} \mathrm{yr}^{-1}$.

changes which do not significantly change the general structure of the disc.

\subsection{Vaporization of the pristine dust component}

The annealed pristine dust components olivine, pyroxene, and quartz evaporate as they are moved into warm inner disc zones. The elements $\mathrm{Si}, \mathrm{Mg}$, Fe (and O) liberated by this process either form new dust components at low temperatures or form molecular or atomic species in the gas-phase at higher temperatures. The radial distribution of the elements liberated by the evaporation of the pristine dust components is described by Eqs. (22), (36), and (39). Figure 7 shows the resulting radial variation of the abundances of the main dust forming elements $\mathrm{Si}, \mathrm{Mg}, \mathrm{Fe}$ (and $\mathrm{O}$ ) not bound in the ISM dust component, obtained by our computation of the disc structure and chemistry. A conspicuous feature of the resulting abundance distributions is the enrichment of these elements in the region of dust evaporation over their abundances in the pristine matter falling from the parent molecular cloud onto the accretion disc. This results from the diffusive transport of vapours from the evaporation zone into cooler regions, opposing the effect of inwards transport by accretion. This local enrichment of elements is already predicted by the analytical studies of Morfill \& Völk (1984) and Morfill et al. (1985) and our numerical results even resemble their semi-analytically calculated abundance profiles.

\subsection{Iron grains}

Figure 8 shows the relative deviation of the true gas-phase abundance of $\mathrm{Fe}$ atoms from the concentration of the partial pressure of $\mathrm{Fe}$ in the gas-phase if this equals the vapour pressure of iron. In Sect. 5.4.4 we have argued that the deviations from equilibrium are always very small and for most purposes one can assume that the $\mathrm{Fe}$ concentration equals its equilibrium value.

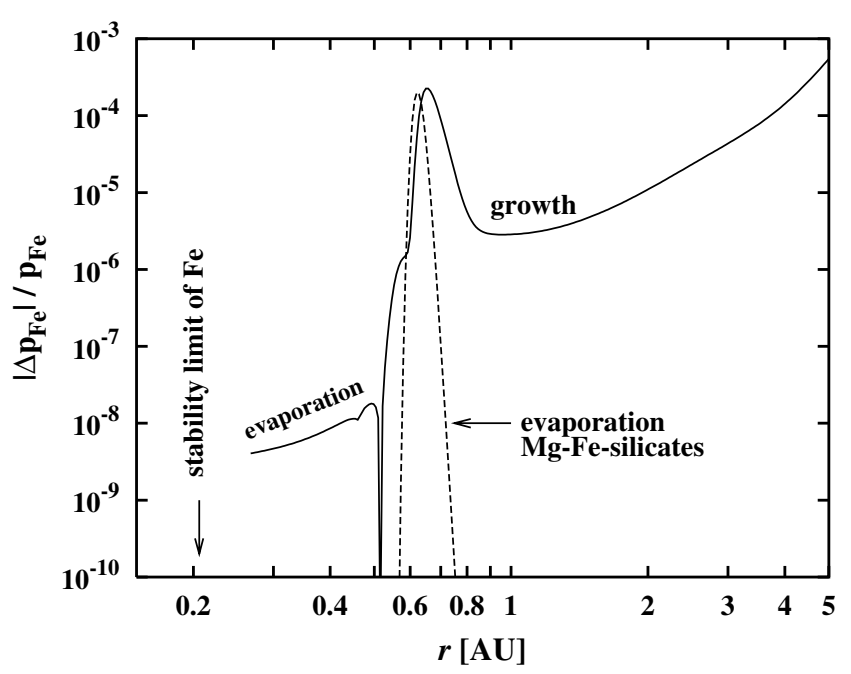

Fig. 8. Relative deviation of the Fe concentration in the gas-phase from the equilibrium concentration for the model with accretion rate $\dot{M}=$ $1 \times 10^{-7} M_{\odot} \mathrm{yr}^{-1}$. In the region $r>0.5$ AU labeled by "growth", the gas-phase concentration of $\mathrm{Fe}$ atoms exceeds that of the equilibrium vapour pressure, in the region labeled by "evaporation" it is less than the equilibrium concentration. The dashed line shows the evaporation rate of the $\mathrm{Mg}$-Fe-silicates in arbitrary units.

The actual concentration of $\mathrm{Fe}$ in the gas-phase, calculated by solving the differential Eq. (26), exceeds the equilibrium concentration in the region outwards of about $r=0.5 \mathrm{AU}$. This region is labeled by "growth" in Fig. 8 since the iron grains increase in size if $c_{\mathrm{Fe}}>c_{\mathrm{Fe} \text {,eq }}$. The actual concentration is less than the equilibrium concentration inside of the radius $r=0.5 \mathrm{AU}$. This region is labeled by "evaporation" in Fig. 8 since the iron grains decrease in size if $c_{\mathrm{Fe}}<c_{\mathrm{Fe}, \text { eq }}$. The difference has a local maximum close to the radius where the evaporation rate of olivine and pyroxene peaks. This evaporation introduces free iron atoms into the gas-phase which are distributed outwards and inwards by diffusional mixing until in the outer region the iron vapour has completely precipitated onto the iron grains.

The relative deviations between the actual concentration and the equilibrium concentration of $\mathrm{Fe}$ in the gas-phase are very small in both cases. In the innermost disc region the difference between the actual $\mathrm{Fe}$ concentration determined from Eq. (26) and the equilibrium concentration is in fact so small that it could not be calculated with sufficient accuracy. For $r \lesssim$ $0.35 \mathrm{AU}$ the difference disappears in the numerical errors of the calculation and it is set to zero in this region.

Obviously the numerical results for the deviation of the partial pressure of iron vapour from its chemical equilibrium value are fully in accord with the predictions of the simple estimates of Sect. 5.4.4.

Since the deviations between the true and the chemical equilibrium partial pressure of $\mathrm{Fe}$ are extremely small the degree of condensation of $\mathrm{Fe}$ into solid iron can be calculated with good accuracy as for a chemical equilibrium state. For the element abundance of $\mathrm{Fe}$ one has to use the abundance of Fe not bound in the pristine dust components, as determined 


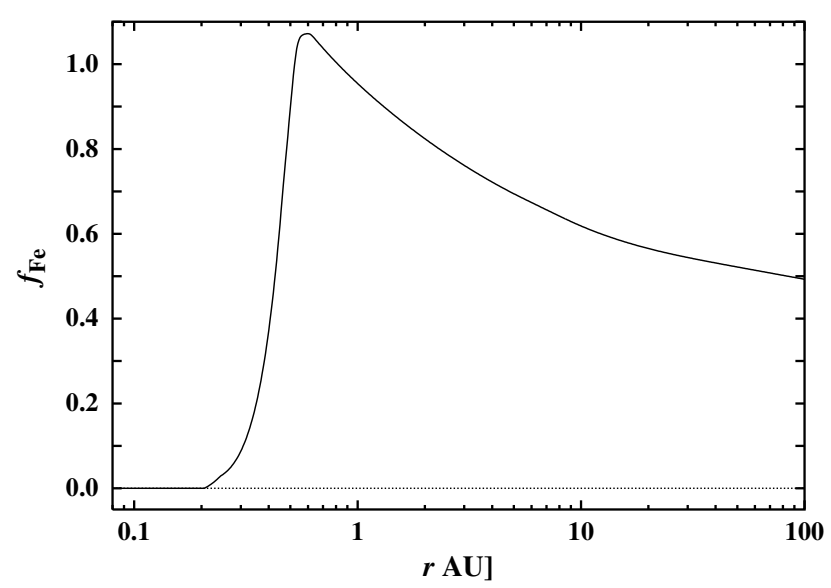

Fig. 9. Radial variation of the fraction $f_{\mathrm{Fe}}$ of $\mathrm{Fe}$ condensed into iron grains, normalized to the solar abundance of $\mathrm{Fe}$. By diffusional mixing the abundance of iron is locally increased over the solar Fe abundance. Disc model with $\dot{M}=1 \times 10^{-7} M_{\odot} \mathrm{yr}^{-1}$.

from Eq. (22) and shown in Fig. 7. Figure 9 shows the resulting radial variation of the degree of condensation of $\mathrm{Fe}$ into iron grains. The abundance of iron grains increases from outside inwards because in the pristine dust mixture part of the iron is bound in olivine- and pyroxene-type silicates. This fraction of the $\mathrm{Fe}$ is liberated in the warm inner disc regions, where the annealed $\mathrm{Mg}$-Fe-silicates evaporate, and the Fe vapour then precipitates on the already existing solid iron. Again, the accumulation of iron within a certain region of the disc due to the outwards transport of vapour from the evaporation zone of iron grains can clearly be seen. This effect is not particular strong, but in any case it is non-negligible.

\subsection{Abundance of the silicate dust species}

The model calculation considers the destruction of the pristine silicate dust components with olivine-, pyroxene-, and quartz-type composition and the formation of the equilibrium dust components forsterite and enstatite.

Figure 10 shows the results for the radial variation of the degree of condensation of the different dust species for a disc model with an accretion rate of $\dot{M}=1 \times 10^{-7} M_{\odot} \mathrm{yr}^{-1}$. It shows the degree of condensation of $\mathrm{Si}$ into the different $\mathrm{Si}$ bearing solid compounds, and the fraction of the Si present as $\mathrm{SiO}$ molecule. As can be clearly seen the pristine olivine-, pyroxene- and quartz-type dust components gradually disappear between the outer disc and a radius of about $0.55 \mathrm{AU}$ which is the point where the last grains of these silicates disappear. The evaporation temperatures of the three species are not identical, but too close to each other for this difference to be recognized in the figure. The annealed olivine, pyroxene and quartz grains disappear at a significantly lower temperature than the equilibrium compounds in the mixture, forsterite and enstatite, because of their lower stability. The continuous decrease of the abundance of the pristine silicates with decreasing radius results from diffusional mixing, which mixes material free of olivine, pyroxene and quartz from the inner disc,

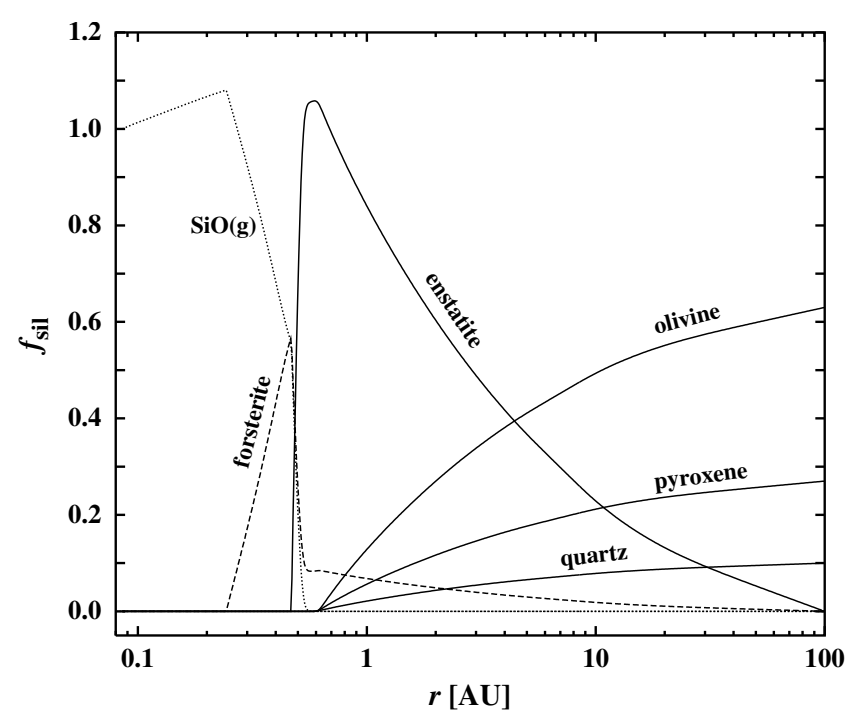

Fig. 10. Radial variation of the fraction of the silicon condensed into the individual Si-bearing condensates and the fraction of silicon present as $\mathrm{SiO}$ molecules, normalized to the solar abundance of Si. By diffusional mixing the abundance of Si-compounds is locally increased over the solar Si abundance. Disc model with $\dot{M}=$ $1 \times 10^{-7} M_{\odot} \mathrm{yr}^{-1}$.

interior to the evaporation limit at about $r=0.55 \mathrm{AU}$, into the outer disc zone and dilutes the unprocessed interstellar material with material from the inner disc region. As a result part of the pristine dust is replaced by condensation products from the inner disc: crystalline forsterite and enstatite.

The figure also shows the fraction of $\mathrm{Si}$ present as $\mathrm{SiO}$ molecules in the gas-phase. The zones where the pristine silicates disappear and the zone of evaporation of the equilibrium components, characterized by the coexistence of solids and a non-negligible fraction of $\mathrm{SiO}$ molecules in the gasphase, obviously are well separated. They occur in different regions of the disc.

Figure 11 again shows the degree of condensation of the silicates, but this time in a cumulative representation which shows how the fractions of the silicon bound in the individual species add up to the total Si abundance. The figure shows the total abundance of silicon bound into dust normalized to the $\mathrm{Si}$ abundance of the material from the parent molecular cloud. The most remarkable feature is that the added-up Si abundance of the silicates exceeds unity. This is a result of the diffusion of solids and vapour, which is predicted in the studies of Morfill (1983) Morfill \& Völk (1984), Morfill (1985), and Morfill et al. (1985). The outwards decrease of the $\mathrm{SiO}$ concentration drives a diffusion current of condensible material inside out where it precipitates onto grains. This leads to an intermediate zone of accumulation of Si-bearing material which corresponds in our own Solar System to the region of the innermost terrestrial planets. The increase of the $\mathrm{Si}$ abundance over its interstellar value is not particularly strong, but on the other hand it is not negligibly small. 


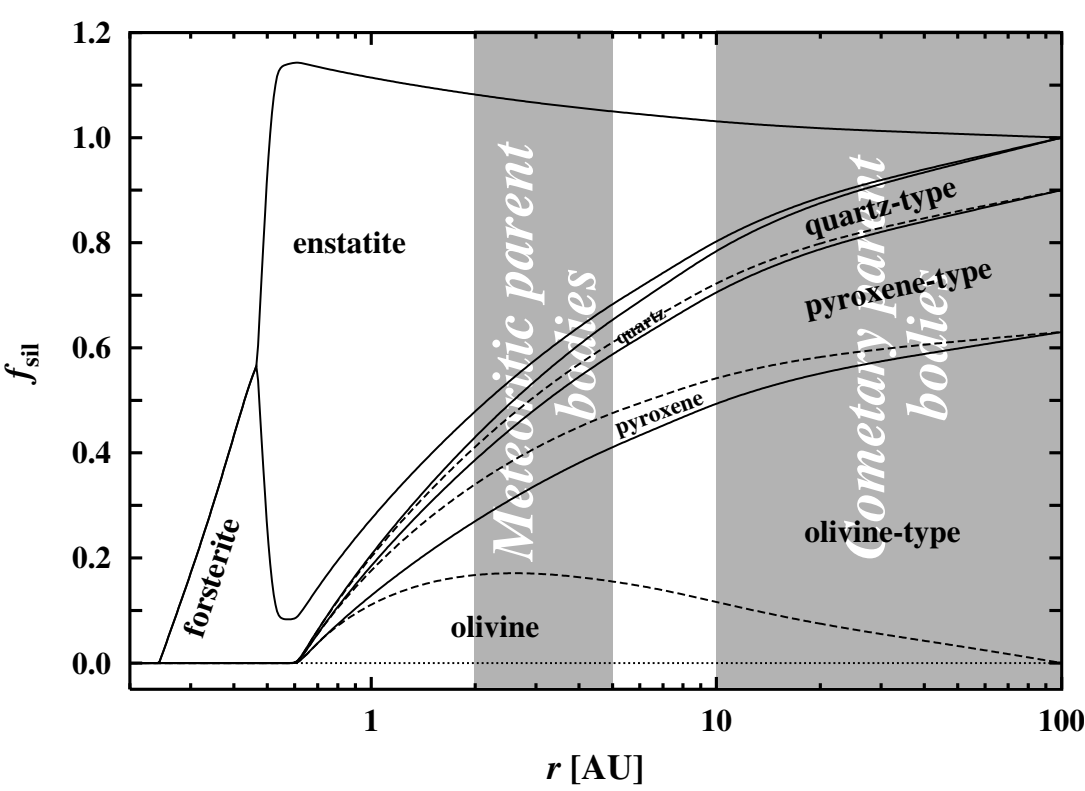

Fig. 11. Cumulative representation of the distribution of the silicon condensed into dust between the different kinds of silicate dust components (interstellar and equilibrium ones) within the protoplanetary disc. The total $\mathrm{Si}$ abundance is normalized to the interstellar Si abundance. An enhancement of the $\mathrm{Si}$ abundance in the region of evaporation of the interstellar silicates is due to diffusive mixing effects. The grey-shaded areas roughly indicate the regions where the parent bodies of meteorites and cometary nuclei are formed. Stationary disc model with an accretion rate $\dot{M}=1 \times 10^{-7} M_{\odot} \mathrm{yr}^{-1}$.

\subsection{Comparison with Solar System bodies}

\subsubsection{Limitations of the results of the model calculation}

The main shortcoming of the present model calculation is that it is performed for a stationary one-zone model, which somewhat restricts the possibilities for a comparison of the results with real accretion discs. A comparison of the present model with the time-dependent model calculation of Paper II shows that the radial distribution of the dust components common to both model calculations are to some extent similar at about $t=10^{6} \mathrm{yrs}$ and out to a distance of roughly $20 \mathrm{AU}$, but that they are not completely equivalent. Beyond $20 \mathrm{AU}$ the results for the stationary and the time-dependent model calculations strongly disagree, because mixing cannot transport matter from the innermost parts of the disc $(r<1 \mathrm{AU})$ significantly beyond 20 AU within $10^{6}$ years (cf. Paper II and Fig. 9 in Paper I). The results of the present calculation, thus, can only be taken as a crude approximation to the radial structure of a protoplanetary disc and the composition of the mineral mixture for the inner disc region up to $\approx 20 \mathrm{AU}$ at several $10^{5} \mathrm{yrs}$.

In the present model calculation turbulent diffusion is considered as the sole process responsible for radial mixing in the disc. Preliminary calculations of time dependent 2-D hydrodynamic disc models (Keller \& Gail 2002; Kley \& Lin 1992) however show that large-scale circulation currents present in protoplanetary discs are at least as important for large-scale radial mixing of the disc material as is turbulent mixing. This additional mixing process is lacking in the present model.

\subsubsection{Composition of meteorite and cometary dust}

As a result of the model calculation one obtains the radial variation of the composition of the mineral mixture in the protoplanetary accretion disc. It is this mixture of silicate dust species which enters the planetesimals during their growth which in turn form the building blocks for the subsequent process of planetary formation. The formation of the planetesimals and the formation of planets from the planetesimals is generally assumed to start several $10^{5}$ yrs after the formation of the protostar (e.g Cameron 1995). We can thus compare the results of the present model calculation to the composition of planetesimals in the formation phase of the Solar System.

In our own Solar System we have two types of objects in which material from the early formation time of the solar system survived:

1) Primitive meteorites, which are debris particles from collisions of undifferentiated asteroids from the asteroid zone of our Solar System between Mars and Jupiter. Besides chondrules and $\mathrm{Ca}-\mathrm{Al}$-inclusions they contain varying percentages of a fine-grained material, called the matrix. The matrix material in primitive meteorites is thought to represent pristine dust from the Solar Nebular (e.g. Scott et al. 1988) which was only slightly processed by thermal and aqueous alteration on the meteoritic parent body. The composition of the matrix material probably closely resembles the composition of the dust from which the planetesimals are made. The chondrules and $\mathrm{Ca}-\mathrm{Al}$-inclusions also are surviving material from the formation phase of the planetary system, they are, however, heavily processed and are not representative for the primordial dust.

2) Cometary nuclei, which are thought either to be surviving planetesimals from the giant planet zone out to Neptune (the long period comets from the Oort cloud) or from the zone beyond Neptune (the short period comets from the Kuiper belt). Since this material was never heated to temperatures higher than about $20 \mathrm{~K}$ during the last $4.6 \mathrm{Gyr}$ until the body entered the inner Solar System, the dust grains in these comets are also thought to represent truly pristine dust from the Solar Nebula.

Table 4 shows the composition of the silicate mixture in our disc model at two radii of $3 \mathrm{AU}$ and $20 \mathrm{AU}$ which are roughly representative for the regions where the parent bodies of meteorites and the cometary nuclei of long period comets, respectively, have been formed. The results outline the trends which 
Table 4. Composition of the silicate mixture at 3 and $20 \mathrm{AU}$ in a stationary protoplanetary disc model with $\dot{M}=1 \times 10^{-7} M_{\odot} \mathrm{yr}^{-1}$. The numbers are fractions of the silicon contained in the different dust species. "cr" and "am" denote crystalline dust or amorphous dust with a composition like that of the indicated crystalline material, respectively.

\begin{tabular}{ccccccccc}
\hline \hline$r$ & \multicolumn{2}{c}{ olivine } & forsterite & \multicolumn{2}{c}{ pyroxene } & \multicolumn{2}{c}{ enstatite } & \multicolumn{2}{c}{ quartz } \\
$\mathrm{AU}$ & $\mathrm{cr}$ & $\mathrm{am}$ & $\mathrm{cr}$ & $\mathrm{cr}$ & $\mathrm{am}$ & $\mathrm{cr}$ & $\mathrm{cr}$ & $\mathrm{am}$ \\
\hline 3 & 0.159 & 0.158 & 0.037 & 0.067 & 0.070 & 0.458 & 0.023 & 0.027 \\
20 & 0.073 & 0.469 & 0.010 & 0.031 & 0.202 & 0.129 & 0.011 & 0.076 \\
\hline
\end{tabular}

are to be expected for the composition of the silicate mineral mixture in these objects:

- A mixture of interstellar silicates and of equilibrated silicate dust from the warm inner disc region in about equal proportions and a rather high degree of crystallization of the interstellar dust in the zone where the parent bodies of meteorites are formed.

- Mostly interstellar dust with an admixture of up to about $20 \%$ annealed interstellar dust and equilibrated dust from the warm inner disc region in the zone where cometary nuclei are formed.

From comets one has a direct indication for the kind of mixing between disc material from the warm inner and cold outer zones, as it is predicted by the model calculation. This indication comes from observations of dust emission from comets where an analysis of the silicate emission bands definitely shows that part of the silicate dust is crystalline. Hanner et al. (1994) discussed in their analysis of comet Mueller 1993a the origin of this crystalline dust component. As there are no indications for significant amounts of crystalline silicate dust to be present in the interstellar medium and in molecular clouds, the crystalline dust is likely to originate within the Solar Nebula itself. It can result either from annealing of amorphous dust or by growth of crystalline grains from the gas-phase. Both processes require elevated temperatures ( $T \gtrsim 800 \mathrm{~K}$ in our model calculations) which are only encountered in the inner part of the solar nebula $(r \lesssim 1 \ldots 2 \mathrm{AU})$, but not in the region beyond Jupiter where comets are thought to form. This suggests that some radial mixing took place between the inner and outer parts of the Solar Nebula. Wooden et al. $(1999,2000)$ discussed the structure of the silicate band in comet Hale-Bopp and showed that the cometary material contains a considerable fraction of crystalline silicates, probably with quite a high magnesium content which is characteristic for chemical equilibrium condensates at high temperature (e.g. Saxena \& Ericksson 1986). This indicates mixing of material from high temperature regions of the disc to the formation zone of cometary bodies. Another explanation may be that this material is of circumstellar origin from e.g. AGB stars, as is favored by Wooden et al. (1999); this, however, immediately raises the question why no crystalline grains are seen in the ISM.

Nuth (1999) and Nuth et al. (2000) interpreted the presence of crystalline material in comets as an indication of radial transport of matter from warm inner regions of a protostellar accretion disk where annealing of initially amorphous grains is possible to the cold outer zones of the disk. They point out, that possibly even an evolutionary sequence is seen in disks around pre-main-sequence A and B stars showing an increase of the amount of crystalline material present with age of the objects, which may result from progressive mixing.

For meteoritic matrix material the fraction of enstatite in the mixture predicted by the model calculation seems to be somewhat high, but the general trends fits the observed composition of the matrix material of primitive meteorites (e.g. Scott et al. 1988; Brearley et al. 1989; Buseck \& Hua 1993); however, even for the most primitive meteorites the material is not truly pristine since it shows clear indications of chemical alterations (cf. Wasson 1985).

The high enstatite content in the model may result from the present assumption of complete chemical equilibrium between forsterite and enstatite, which possibly cannot be attained because of the slow forsterite-enstatite conversion (cf. Fig. 5). A realistic modeling of the kinetics of the forsterite-enstatite interconversion likely reduces the fraction of enstatite in the mixture.

In principle the interplay between radial transport processes in the disc, the annealing of amorphous ISM dust, and the evaporation and re-condensation processes, as considered in the present model calculation, seems to be able to reproduce some of the basic properties of the most pristine relic materials in our Solar System backdating to the earliest formation period of the planetary system. This demonstrates the important role that large-scale radial mixing processes have played for the composition of the protoplanetary disc and explains the presence of high-temperature equilibrated material in low-temperature regions of the early Solar System.

\section{Concluding remarks}

In this paper the basic set of equations for describing the dust metamorphosis from the interstellar dust mixture of the parent molecular cloud to a chemical equilibrium composition have been worked out. The equations have been solved selfconsistently together with the equations for a stationary onezone accretion disc model in order to get an impression how the dust metamorphosis in an accretion disc proceeds.

The results show that this simple model can successfully reproduce some basic properties of the composition of the most primitive materials we have in our Solar System. A detailed comparison of the results of such a calculation, however, will be possible only if at least two-dimensional time-dependent 
models for the turbulent (MHD) flows in accretion discs become available, because one-zone models cannot properly account for the probably very important effects of vertical mixing in the disc. Cylindrically symmetric, time-dependent 2-D calculations for the hydrodynamics, chemistry, and radiative transfer in protoplanetary discs are presently under way. Additionally, some conversion processes between different dust materials, especially the pyroxene-enstatite conversion, have to be modeled more accurately than has been done in this paper. Also a number of additional materials not considered in this model calculation has to be added to the model calculation in order to improve the modeling, especially the $\mathrm{Al}$ and $\mathrm{Ca}$ compounds and the iron oxides and sulfides.

Acknowledgements. I thank the referee A.P. Jones for carefully reading the manuscript and many suggestions to improve the presentation and the editor J. Katgert for improving the language. This work is part of a project of the special research project SFB 359 "Reactive Flows, Diffusion and Transport" which is supported by the Deutsche Forschungsgemeinschaft (DFG).

\section{References}

Anders, E., \& Grevesse, N. 1989, Geochim. Cosmochim. Acta, 53, 197

Barin, I. 1995, Thermochemical Data of Pure Substances, 3rd ed. (Weinheim: VCH Verlagsgesellschaft)

Beckwith, S. V. W., \& Sargent, A. I. 1993, in Protostars \& Planets III, ed. E. H. Levy, \& J. I. Lunine (Tucson: University of Arizona Press), 521

Bockelée-Morvan, D., Gautier, D., Hersant, F., Huré, J.-M., \& Robert, F. 2002, A\&A, 384, 1107

Bouwman, J., Meeus, G., de Koter, A., et al. 2001, A\&A, 375, 950

Bradley, J. P. 1994, Science, 265, 925

Brearley, A. J., Scott, E. R. D., Keil, K., et al. 1989, Geochim. Cosmochim. Acta, 53, 2081

Buseck, P. R., \& Hua, X. 1993, Ann. Rev. Earth Planet. Sci., 21, 255

Calvet, N., Hartmann, L., \& Strom, S. E. 2000, in Protostars \& Planets IV., ed. V. Mannings, A. P. Boss, \& S. S. Russell (Tucson: University of Arizona Press), 337

Cameron, A. G. W. 1995, Meteoritics, 30, 133

Chakraborty, S. 1997, J. Geophys. Res., 102, 12317

Chase Jr., M. W., Davies, C. A., Downey Jr., J. R., et al. 1985, JANAF Thermodynamic Tables (Gaithersburg: National Institute of Standards and Technology)

Choi, B.-G., Wasserburg, G. J., \& Huss, G. R. 1999, ApJ, 522, L133

Draine, B. T. 1985, ApJS, 57, 587

Duschl, W. J., Gail, H.-P., \& Tscharnuter, W. M. 1996, A\&A, 312, 624

Ebel, D. S., \& Grossman, L. 2001, Geochim. Cosmochim. Acta, 65, 469

Fabian, D., Jäger, C., Henning, Th., Dorschner, J., \& Mutschke, H. 2000, A\&A, 364, 282

Ferrarotti, A. S., \& Gail, H.-P. 2001, A\&A, 371, 133

Gail, H.-P. 1998, A\&A, 332, 1099

Gail, H.-P. 2001, A\&A, 387, 192 (Paper I)

Gail, H.-P. 2003, in Astromineralogy, ed. Th. Henning (Heidelberg: Springer ), Lect. Notes Phys., 609, 55

Gail, H.-P., \& Sedlmayr, E. 1999, A\&A, 347, 594

Goodman, A. A., \& Whittet, D. C. B. 1995, ApJ, 455, L181
Grevesse, N., \& Noels, A. 1993, in Origin and Evolution of the Elements, ed. N. Prantzos, E. Vangioni-Flam, \& M. Cassé (Cambridge: Cambridge University Press), 14

Grossman, L. 1972, Geochim. Cosmochim. Acta, 36, 597

Haisch, K. E., Lada, E. A., \& Lada, C. J. 2001, ApJ, 553, L153

Hallenbeck, S. L., Nuth III, J. A., \& Daukantas, P. L. 1998, Icarus, 131, 198

Hallenbeck, S. L., Nuth III, J. A., \& Nelson, R. N. 2000, ApJ, 535, 247

Hanner, M. S., Hackwell, J. A., Russel, R. W., \& Lynch, D. K. 1994, Icarus, 112, 490

Hartmann, L. 2000, Space Sci. Rev., 92, 55

Hashimoto, A. 1990, Nature, 347, 53

Holweger, H. 2001, Joint SOHO/ACE workshop, Solar and Galactic Composition, AIP Conf. Proc., 598, 23

Honda, M., Kataza, H., Okamoto, Y. K., et al. 2003, ApJ, 585, L59

Howk, J. C., Savage, B. D., \& Fabian, D. 1999, ApJ, 525, 253

Huffman, D. R., \& Stapp, J. L. 1973, in Interstellar Dust and Related Topics, ed. J. M. Greenberg, \& H. C. van de Hulst (Dordrecht: Reidel), Proc. IAU Symp., 52, 297

Jones, A. P. 2000, J. Geophys. Res., 105, 10257

Keller, C., \& Gail, H.-P. 2003, A\&A, submitted

Keller, L. P., Hony, S., Bradley, J. P., et al. 2003, Nature, 417, 148

Kley, W., \& Lin, D. N. C. 1992, ApJ, 397, 600

Lattimer, J. M., Schramm, D. N., \& Grossman, L. 1978, ApJ, 219, 230

Lauretta, D. S., Kremser, D. T., \& Fegley Jr., B. 1996, Icarus, 122, 288

Lauretta, D. S., Lodders, K., \& Fegley Jr., B. 1998, Meteor. Planet. Sci., 33, 821

Li, A., \& Greenberg, J. M. 1997, A\&A, 323, 566

Lide, R. D. 1995, CRC Handbook of Chemistry and Physics, 76th ed. (Boca Raton: CRC Press)

Lin, D. N. C., \& Papaloizou, J. C. B. 1996, ARA\&A, 34, 703

Martin, P. G. 1995, ApJ, 445, L63

Mathis, J. S. 1996, ApJ, 472, 643

Mathis, J. S., Rumpl, W., \& Nordsieck, K. H. 1977, ApJ, 217, 425

Morfill, G. E. 1983, Icarus, 53, 41

Morfill, G. E. 1985, in Birth and Infancy of Stars, ed. R. Lucas, A. Omont, \& R. Stora (Elsevier Science Publishers), 693

Morfill, G. E., \& Völk, H.-J. 1984, ApJ, 287, 371

Morfill, G. E., Tscharnuter, W. M., \& Völk, H.-J. 1985, in Protostars \& Planets II, ed. D. C. Black, \& M. S. Matthews (Tucson: University of Arizona Press), 493

Nagahara, H., Kushiro, I., \& Mysen, B. O. 1994, Geochim. Cosmochim. Acta, 58, 1951

Nagahara, H., \& Ozawa, K. 1994, Meteoritics, 29, 508

Nagahara, H., \& Ozawa, K. 1996, Geochim. Cosmochim. Acta, 60, 1445

Nichols Jr., R. H., \& Wasserburg, G. J. 1995, Lunar \& Planetary Sci. Conf., XXVI, 1047

Nichols Jr., R. H., Grimley, R. T., \& Wasserburg, G. J. 1998, Meteor. Planet. Sci., 33, A115

Nittler, L. R., Alexander, C. M. O’D., Gao, X., Walker, R. M., \& Zinner, E. 1997, ApJ, 483, 475

Nuth, J. A. 1999, Lunar and Planetary Sci. Conf., 30, 1726

Nuth, J. A., Hill, H. G. M., \& Kletetschka, G. 2000, Nature, 406, 275

Pollack, J. B., Hollenbach, D., Beckwith, S., et al. 1994, ApJ, 421, 615

Prinn, R. G., \& Fegley Jr., B. 1989, in Origin and Evolution of Planetary and Satellite Atmospheres, ed. S. K. Atreya, J. B. Pollak, \& M. S. Mathews (Tucson: University of Arizona Press), 78

Ruden, S. P., \& Pollack, J. B. 1991, ApJ, 375, 740

Saxena, S. K., \& Eriksson, G. 1986, in Chemistry and Physics of Terrestrial Planets, ed. S. K. Saxena (New York: Springer), 30

Schwandt, C. S., Cygan, R. T., \& Westrich, H. R. 1998, Contrib. Mineral. Petrol., 130, 390 
Scott, E. R. D., Barber, D. J., Alexander, C. M., Hutchinson, R., \& Peck, J. A. 1988, in Meteorites and the Early Solar System, ed. J. F. Kerridge, \& M. S. Matthews (Tucson: University of Arizona Press), 718

Sharp, C. M., \& Huebner, W. M. 1990, ApJS, 72, 417

Stahler, S. W., \& Walter, F. M. 1993, in Protostars \& Planets III, ed. E. H. Levy, \& J. I. Lunine (Tucson: University of Arizona Press), 405

Stepinski, T. F. 1998, ApJ, 507, 361

Steyer, T. 1974, Thesis, University of Arizona

Tachibana, S., \& Tsuchiyama, A. 1998, Geochim. Cosmochim. Acta, 62,2005

Tachibana, S., Tsuchiyama, A., \& Nagahara, H. 2002, Geochim. Cosmochim. Acta, 66, 713
Tsuchiyama, A., Tachibana, S., \& Takahashi, T. 1999, Geochim. Cosmochim. Acta, 63, 2451

Wasson, J. T. 1985, Meteorites: Their Record of Early Solar-System History (New York: Freeman \& Comp.)

Wehrstedt, M., \& Gail, H.-P. 2002, A\&A, 385, 181 (Paper II)

Wehrstedt, M., \& Gail, H.-P. 2003, A\&A, 410, 917

Weidenschilling, S. J., \& Cuzzi, J. N. 1993, in Protostars and Planets III, ed. E. H. Levy, \& J. I. Lunine (Tucson: University of Arizona Press), 1031

Weingartner, J. C., \& Draine, B. T. 2001, ApJ, 548, 296

Wooden, D. H., Harker, D. E., Woodward, C. E., et al. 1999, ApJ, 517, 1034

Wooden, D. H., Butner, H. M., Harker, D. E., \& Woodward, C. E. 2000, Icarus, 143,126 